\title{
Recognition of COVID-19 from CT Scans Using Two-Stage Deep-Learning-Based Approach: CNR-IEMN ${ }^{\dagger}$
}

\author{
Fares Bougourzi ${ }^{1}\left(\mathbb{D}\right.$, Riccardo Contino $^{1}$, Cosimo Distante $^{1,2, *(\mathbb{C})}$ and Abdelmalik Taleb-Ahmed ${ }^{3(\mathbb{C}}$ \\ 1 Institute of Applied Sciences and Intelligent Systems, National Research Council of Italy, 73100 Lecce, Italy; \\ fares.bougourzi@isasi.cnr.it (F.B.); riccardo.contino@isasi.cnr.it (R.C.) \\ 2 Department of Innovation Engineering, University of Salento, 73100 Lecce, Italy \\ 3 Univ. Polytechnique Hauts-de-France, Univ. Lille, CNRS, Centrale Lille, UMR 8520, \\ F-59313 Valenciennes, France; Abdelmalik.Taleb-Ahmed@uphf.fr \\ * Correspondence: cosimo.distante@cnr.it \\ $+\quad$ This paper is an extended version of our conference paper: Bougourzi, F., Contino, R., Distante, C., \\ Taleb-Ahmed, A. CNR-IEMN: A Deep Learning Based Approach to Recognise COVID-19 from CT-Scan. \\ In Proceedings of the ICASSP 2021-2021 IEEE International Conference on Acoustics, Speech and Signal \\ Processing (ICASSP), Toronto, ON, Canada, 6-11 June 2021.
}

Citation: Bougourzi, F.; Contino, R.; Distante, C.; Taleb-Ahmed, A.

Recognition of COVID-19 from CT

Scans Using Two-Stage

Deep-Learning-Based Approach:

CNR-IEMN. Sensors 2021, 21, 5878.

https://doi.org/10.3390/s21175878

Academic Editor: Christoph M. Friedrich

Received: 20 July 2021

Accepted: 29 August 2021

Published: 31 August 2021

Publisher's Note: MDPI stays neutral with regard to jurisdictional claims in published maps and institutional affiliations.

Copyright: (c) 2021 by the authors. Licensee MDPI, Basel, Switzerland. This article is an open access article distributed under the terms and conditions of the Creative Commons Attribution (CC BY) license (https:// creativecommons.org/licenses/by/ $4.0 /)$.

\begin{abstract}
Since the appearance of the COVID-19 pandemic (at the end of 2019, Wuhan, China), the recognition of COVID-19 with medical imaging has become an active research topic for the machine learning and computer vision community. This paper is based on the results obtained from the 2021 COVID-19 SPGC challenge, which aims to classify volumetric CT scans into normal, COVID-19, or community-acquired pneumonia (Cap) classes. To this end, we proposed a deep-learning-based approach (CNR-IEMN) that consists of two main stages. In the first stage, we trained four deep learning architectures with a multi-tasks strategy for slice-level classification. In the second stage, we used the previously trained models with an XG-boost classifier to classify the whole CT scan into normal, COVID-19, or Cap classes. Our approach achieved a good result on the validation set, with an overall accuracy of $87.75 \%$ and $96.36 \%, 52.63 \%$, and $95.83 \%$ sensitivities for COVID-19, Cap, and normal, respectively. On the other hand, our approach achieved fifth place on the three test datasets of SPGC in the COVID-19 challenge, where our approach achieved the best result for COVID-19 sensitivity. In addition, our approach achieved second place on two of the three testing sets.
\end{abstract}

Keywords: deep learning; multi-tasks strategy; slice-level classification; COVID-19; CT scans

\section{Introduction}

COVID-19 is a respiratory infection caused by the virus named SARS-CoV-2 belonging to the coronavirus family. The virus mainly affects the respiratory tract but can also cause symptoms affecting other organs [1]. In more than half of the cases, the infection is asymptomatic, and in about a third of the cases, it presents flu-like symptoms [2]. Since the appearance of COVID-19 in Wuhan, China, in 2019, much effort has been made to fend off the spreading of COVID-19 infection. To stop the spread of COVID-19, it is mandatory to recognize and then confine infected persons. Many recognition methods have proved their efficiency including RT-PCR, CT scans, and X-ray scans [3]. Despite the fact that the RT-PCR test is considered as the gold standard in diagnosing COVID-19, it has a considerable falsenegative rate, especially in early stages of infection [4]. In contrast, using X-ray scan and CT scan methods can give efficient results in both time and accuracy [3]. In fact, using CT scans and X-ray scans requires an expert radiologist to identify the COVID-19 infection. Artificial intelligence (AI) systems can provide an alternative solution for automatic diagnosis of COVID-19 infections [3,5].

This paper is an extended version for our IEEE International Conference on Acoustics, Speech, and Signal Processing (ICASSP) paper [6] for the 2021 COVID-19 SPGC challenge [7]. The objective of this work is to give more details about each step of our proposed 
approach. Furthermore, we highlight all experiments that we have conducted in developing our approach. In addition, we discuss the results of each experiment to provide more understanding of our work. To have more insight into the trained CNN models, we used heat maps to check if the trained models were looking in the right places in the CT scan slices to classify them.

To evaluate the performance of our approach, we used the SPGC-COVID dataset [8], which contains volumetric chest CT scans of 171 patients positive for COVID-19, 60 with community-acquired pneumonia (CAP), and 76 normal cases. In addition to the train and val splits, there are three testing splits with a total of 90 CT scans, and each testing set has specific characteristics and challenges.

To classify the CT scans into COVID-19, CAP, and normal, we proposed an ensemble deep learning approach. Our CNR-IEMN approach consists of two stages. In the first stage, we fine-tuned deep learning models using a multi-tasks strategy for slice-level classification. In the second stage, the entire volume of the CT scan is classified using slice-level predictions and the XG-boost classifier. In summary, the main contributions of this paper are:

- We propose to segment the lung lobes of the slice, and then we stack the original slice channel with the segmented channels to obtain an RGB-like image.

- With the use of the multi-tasks strategy and data augmentation for COVID-19 and Cap slices, we used transfer learning to train four convolutional neural network (CNN) architectures (ResneXt-50, Densenet-161, Inception-v3, and Wide-Resnet-50) for slice-level classification.

- $\quad$ For patient-level CT scan classification, we propose to divide the CT scan slices into groups and then calculate the percentage of each class within each group using all trained CNN models for the slice-Level classification. In the end, we combined all grouping percentages from all trained CNN architectures and then fed them into an XG-boost classifier [9] to recognize the CT scan category.

This paper is organized in following way: Section 2 describes the related works. Section 3 contains the description of the materials and methods used. The experiments, results, and discussion are given in Section 4. Finally, we conclude our paper in Section 5.

\section{Related Works}

Since the appearance of COVID-19, CT scans have been widely used not only to recognize infected patients with COVID-19 but also to quantify the infection and monitor the evolution of the disease $[5,10]$. In the computer vision community, many approaches and frameworks have been proposed to help in recognizing and monitoring COVID-19 infection [5]. Since the deep learning approach has proved its efficiency in most computer vision tasks [11] (including medical imaging tasks [3,12]), most of the approaches that have been proposed for COVID-19 analysis from CT scans are CNN-based approaches. In general, the approaches that use CT scans for COVID-19 analysis can be classified into: segmentation [13-15] and recognition [16-20] approaches.

In [13], Q. Yan et al. proposed a feature variation block which adaptively adjusts the global properties of the features for segmenting COVID-19 infection. Their proposed feature variation block can enhance the capability of feature representation effectively. In addition, they fused features at different scales by proposing Progressive Atrous Spatial Pyramid Pooling to handle the sophisticated infection areas with diverse appearance and shapes. D. Müller et al. [14] proposed an automatic segmentation framework for COVID-19 infection segmentation from limited training data. In their approach, they investigated several preprocessing methods and exploiting extensive data augmentation. Moreover, they implemented a 3D U-Net architecture for both lungs and COVID-19infected region segmentation.

In [16], H. Alshazly et al. investigated different deep learning architectures to classify CT scan images into COVID-19/non-COVID-19 using transfer learning and the LAMB optimizer on two databases. Furthermore, they provided visual explanations of the their 
trained models for detecting COVID-19-infected regions and compared them with expert radiologist annotations. N. Lassau et al. used 58 clinical and biological variables and chest CT scan data to predict the COVID-19 severity score [19]. They trained a CNN architecture using the CT scans to predict severity, and then they constructed the multimodal AIseverity score that includes five clinical and biological variables (age, sex, oxygenation, urea, and platelet) in addition to the deep learning model.

In addition to the two main tasks (COVID-19 segmentation and recognition), there are some works that proposed combining both tasks, such as [21,22]. In [21], C. Zheng et al. proposed a weakly supervised deep-learning-based framework that uses volumetric chest CT scans to detect COVID-19. First, the lung regions were segmented using a pre-trained UNet; then, the segmented 3D lung regions were fed into a 3D deep neural network to predict if the CT scan contained COVID-19 infection or not.

In the 2021 COVID-19 SPGC challenge [7], six conference papers were accepted, which corresponded to the approaches that achieved the best results [23-27]. TheSaviours [25] team proposed a two-stage convolutional neural network approach. The IITDelhi team [26] used a residual network at multiple scales to extract features from all the slices of the $\mathrm{CT}$ scan for each patient. Then, they used these features to train a patient-level classifier. The LLSCP team [24] proposed a multi-stage progressive learning strategy based on a 3D ResNet module. The UniSheff_EEE team [27] exploited transfer learning of a 3D Network (3D ResNet-50) to classify the volumetric CT scans. The Bingo team [27] proposed a novel ensemble learning framework with adaptive boosting and dataset clustering algorithms.

\section{Materials and Methods}

\subsection{Database}

The 2021 COVID-19 SPGC challenge [7] provided SPGC-COVID dataset [8] for the evaluation of the participants' approaches. SPGC-COVID dataset [8] contains volumetric chest CT scans of patients positive for COVID-19 infection, community-acquired pneumonia (CAP), and normal patients. Each CT scan consists of all slices of the CT scan in the Digital Imaging and Communications in Medicine (DICOM) format, with a size of $512 \times 512$. The COVID-19 cases were collected from February 2020 to April 2020, whereas CAP cases and normal cases were collected from April 2018 to December 2019 and January 2019 to May 2020, respectively. Diagnosis of COVID-19 infection is based on positive real-time reverse transcription polymerase chain reaction (rRT-PCR) test results, clinical parameters, and CT scan manifestations identified by three experienced thoracic radiologists. It should be noted that each CT scan comes from a different patient, so the number of persons equals the number of $\mathrm{CT}$ scans.

In 2021 COVID-19 SPGC challenge [7], the dataset contains three splits: train, val, and test. The labels of the CT scans are known for the train and val Splits, while the test scan labels are unknown for all participants. The train and val sets were obtained from 307 volumetric chest CT scans (171 patients positive for COVID-19, 60 with communityacquired pneumonia (CAP), and 76 normal cases). A total of $30 \%$ of these 307 CT scans were selected randomly as validation set, and the remaining $70 \%$ were used as training set. All training and validation CT scans were obtained by SIEMENS, SOMATOM Scope scanner with the normal radiation dose and the slice thickness of $2 \mathrm{~mm}$. Besides the patient-level labels, a subset of 55 COVID-19 and 25 CAP cases were analyzed by one radiologist to identify and label slices with evidence of infection.

In addition to the train and val splits, there were three testing sets:

1. Thirty CT scans of COVID-19, CAP, and normal cases from the same center where the training/validation sets were obtained.

2. Thirty low-dose CT scans (LDCT) of COVID-19 and normal cases obtained with slice thickness of $2 \mathrm{~mm}$.

3. Thirty CT scans of COVID-19, CAP, and normal with a history of heart disease or operation in which an abnormal manifestation related to a non-infection disease is 
demonstrated in most (not all) of the cases. This set was obtained with various slice thickness and radiation doses.

\subsection{Segmentation}

For lung segmentation, we used marker-based watershed segmentation [28], which is based on identifying two markers. The internal marker identifies the lung tissue, and the external marker identifies the outside of the region of interest. The internal marker is obtained by thresholding the image and removing all regions and leaving just the biggest one. The external marker is created by morphological dilation of the internal marker with two different iterations and subtracting the results. A watershed marker is created superimposing the two markers with different gray-scale values. To find the precise border of the lung, the marker-based watershed algorithm is applied on the black strip of the watershed marker and the Sobel gradient image of the original scan. In order not to miss lobes located next to the border regions, a black top-hat operation is performed to re-include those areas and areas surrounding the lung hila. Finally, the segmented lungs mask is obtained by holes closing. Figure 1 shows some examples of the lung segmentation for infected slices with COVID-19 and Cap diseases.
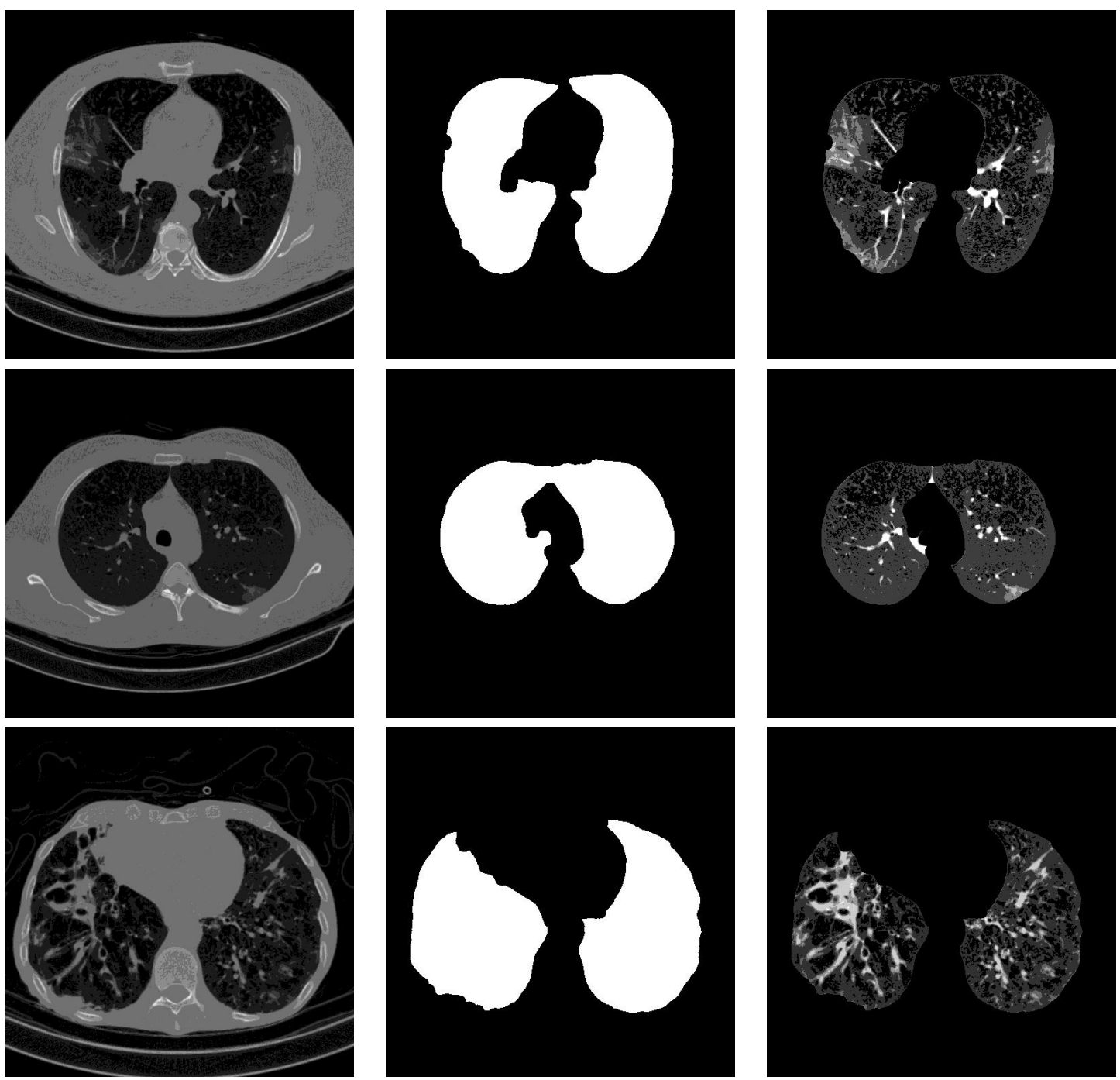

Figure 1. Lung segmentation examples: the first column shows the input CT scan slice, the second column shows the lungs mask result, and the last column shows the lung segmentation results. The corresponding classes for rows 1 to 3 are COVID-19, COVID-19, and Cap, respectively. 


\subsection{Our Proposed Approach}

The overall structure of our approach is summarized in Figure 2. In summary, the proposed approach consists of two main stages. The main focus of the first stage is to classify the slices into one of the three classes (normal, COVID-19, or Cap). To this end, we used the training and validation CT scans that have the slice-level labels for both COVID-19 and Cap classes. Furthermore, we selected the first 30 normal CT scans and labeled all their slices as normal class. Since these CT scans are labeled as normal class, no slice within these CT scans contains COVID-19 or Cap infection. In total, we have 30, 55, and 24 CT scans for normal, COVID-19, and Cap, respectively. These CT scans were used to train and test deep learning architectures for slice-level classification. Among these CT scans, we have 9,17 , and 8 validation CT scans that have the slice level labeling. In the second stage, we predicted the slice label for the whole CT scan using trained CNN architectures. Since the number of slices of the CT scans varies from one CT scan to another, we propose dividing the slices into 20 equal groups. For each group, we calculate the slice prediction percentage of each class. By concatenating the percentages of all CT scan groups, we create a feature vector, which will be fed into an XG-boost classifier [9] to predict the class of the CT scan.

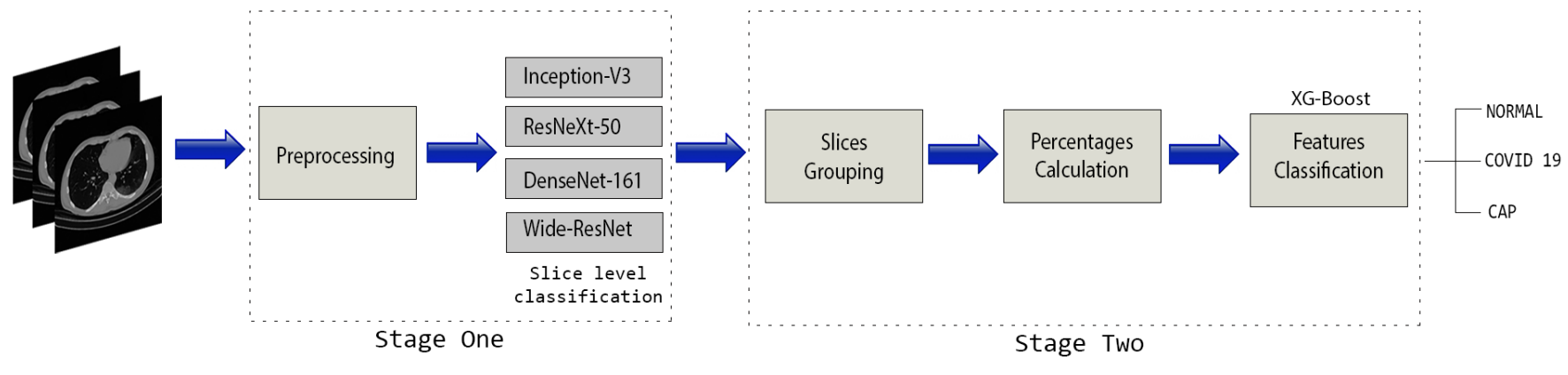

Figure 2. The overall structure of our proposed CNR-IEMN approach [6].

\subsubsection{Slice-Level Classification Stage}

The aim of this stage is to fine-tune the CNN pretrained models to classify the CT scan slices into normal, COVID-19, or Cap class. The slice-level classification is the most important stage in our approach which can influence the whole CT scan prediction.

In the preprocessing phase, we read the slice images from the ".dcm" file format which gives one channel image (grayscale), where we did not use any contrast-enhancement technique. Since most CNN architectures were designed for color images, we propose stacking the gray image, the segmented lung lobes image, using the proposed method in [28] and the multiplication of the grayscale image with the binary lung lobes mask. Figure 3 shows an example of the original image, the segmentation result, and the result of stacking the three channels. In addition to having three channels as input to the CNN architectures, the segmentation guides the slices' classification by concentrating on the lung lobes' features and removing non-relevant ones.

To train the slice-based classification, we have 10,294, 2482, and 742 slices for normal, COVID-19, and Cap classes, respectively. From the number of slices of each class, we notice they are not balanced. To deal with this issue, we used data augmentation techniques for the COVID-19 and Cap slices. The data augmentation techniques used are:

- $\quad$ Color jitter with brightness $=0.2$, contrast $=0.2$, and applying probability $=0.2$.

- $\quad$ Random horizontal flip with applying probability $=0.2$.

- $\quad$ Random perspective with distortion scale $=0.5$.

- Random rotation from -30 to 30 degrees.

- Random cropping. 


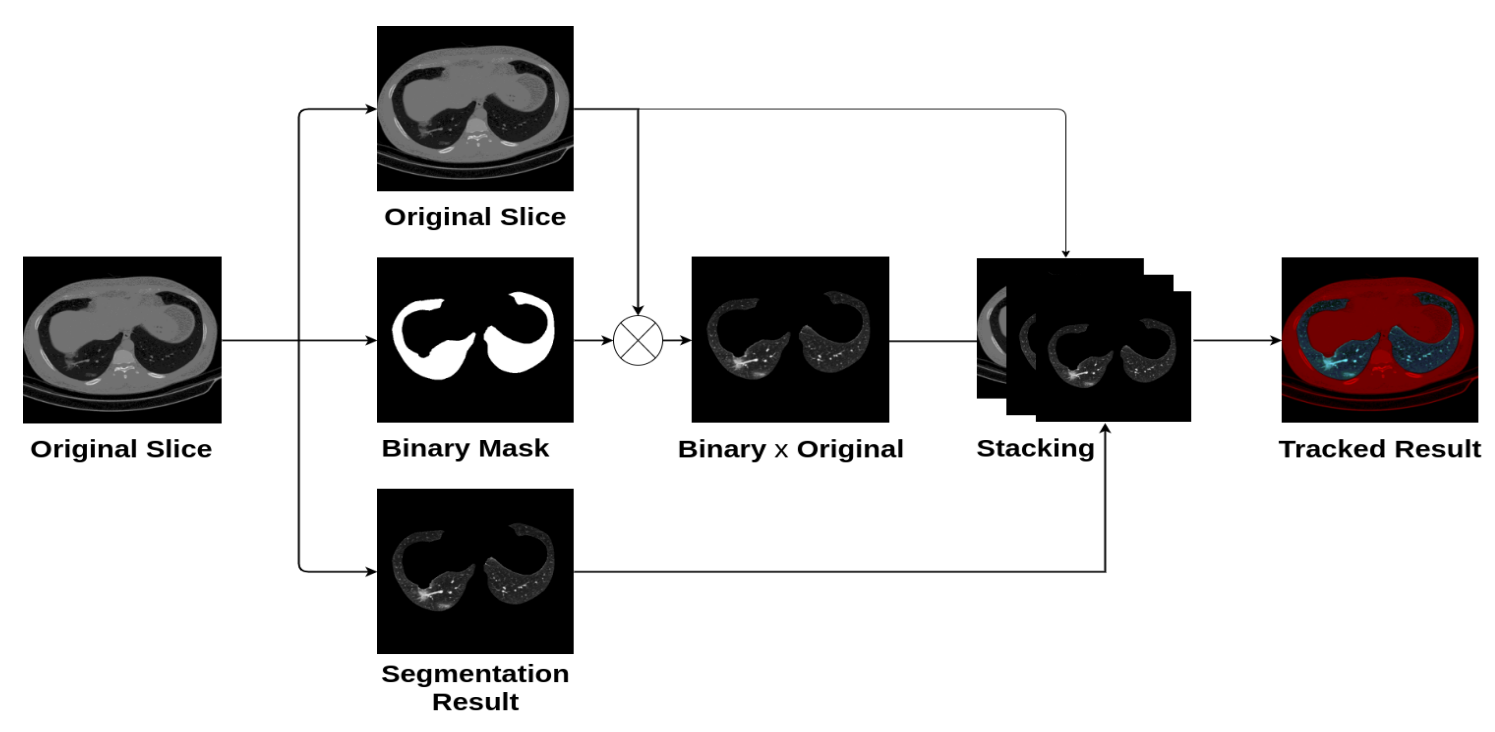

Figure 3. Preprocessing phase.

For COVID-19 class, we generated 3 augmented images from each slice. On the other hand, we generated 10 augmented images for each Cap slice. In total, we obtained 9928 and 8162 for COVID-19 and Cap, respectively.

Figure 4 shows an example of how we trained the four backbone CNN architectures, which are: ResneXt-50 [29], Densenet-161 [30], Inception-v3 [31], and Wide-Resnet-50 [32] (the backbone $\mathrm{CNN}$ architectures are the pre-trained models that were trained on ImageNet challenge database [33]). In our approach, we trained all of the CNN architectures using multi-tasks strategy. In more detail, we fine-tuned the CNN pretrained models, where just the FC layers were randomly initialized using uniform distribution initialization. The first task of the multi-tasks training is the classification of the input image into normal, COVID-19, and Cap classes. Since the size and the shape of the lungs change from the first, middle, and last slices of the CT scan, we divide each CT scan into five equalized regions as shown in Figure 5. The slices within the same region in the CT scan will have the same label. In addition to the main task (to classify the slices into normal, COVID-19, and Cap), the second task classifies the slice into one of the region classes. For the same purpose, we add the total number of slices of the CT scan and the slice location in the CT scan into the deep features (FC layer) as shown in Figure 4.

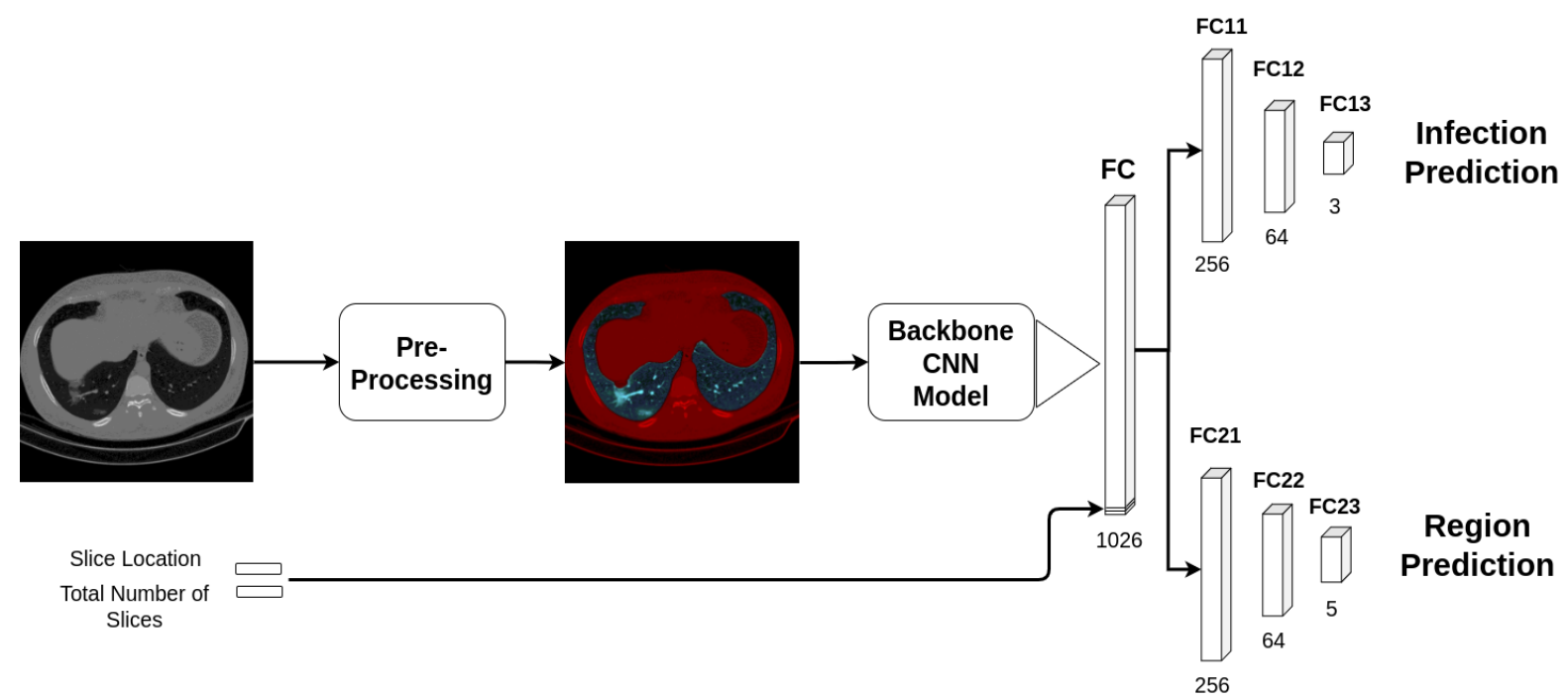

Figure 4. Multi-tasks slice-level training using CNN architectures. 


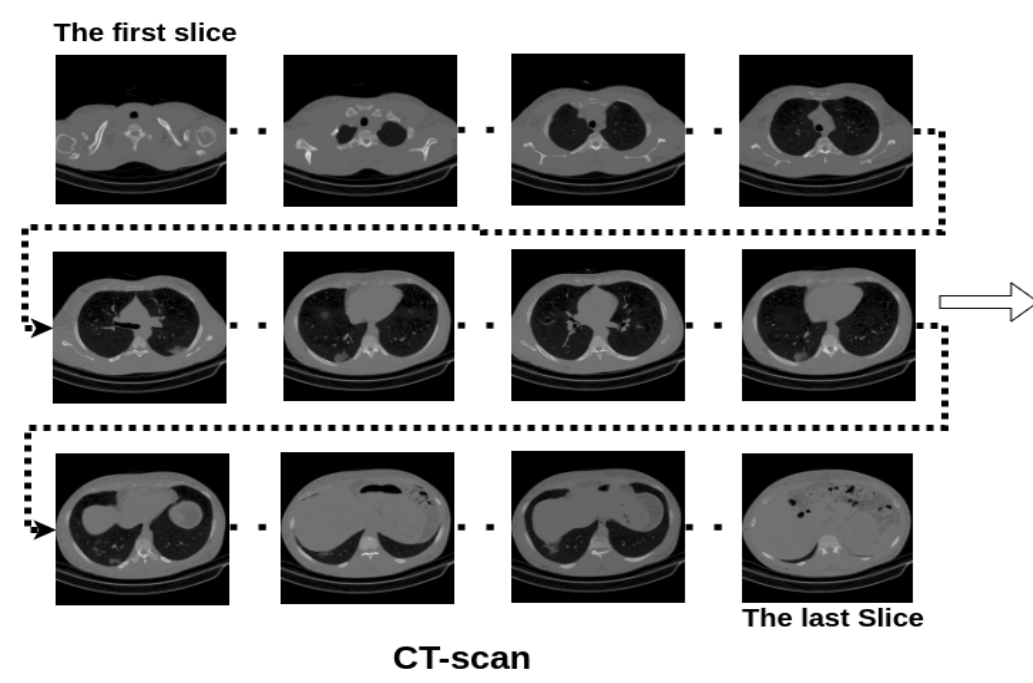

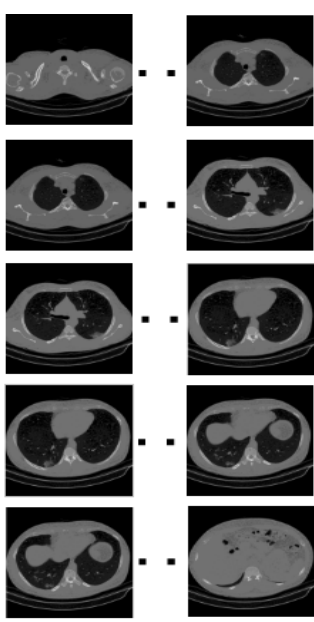

Region 1

Region 2

Region 3

Region 4

The divided five regions

Figure 5. Example of dividing a CT scan into five regions.

\subsubsection{Patient-Level Classification Stage}

Since the CT scans have different number of slices, we propose dividing the CT scan into 20 groups where each group contains the same number of slices. After that, we applied our trained slice-level architectures to predict the labels of the slices of each group; then, we calculate the predicted slices percentage of each class within the group. This produces $20 \times 3=60$ features for each CT scan using one of the trained CNN architectures. For classifying the CT scans, we feed the combination of all trained CNN architecture features, by concatenating them alongside each other, into an XG-boost classifier [9]. In our experimental part, we trained the XG-boost with the training CT scan features; then, we evaluated the performance of our approach using the validation CT scan features.

\section{Results and Discussion}

\subsection{Experimental Setup}

For deep learning training and testing, we used the Pytorch [34] library with NVIDIA GPU Device GeForce TITAN RTX 24 GB. The batch size used consists of 64 images, and as a loss function, we used the focal loss function [35] with gamma equal to 1.5.

\subsection{Slice-Level Classification}

For slice-level classification, four powerful CNN architectures were used: ResneXt-50, Densenet-161, Inception-v3, and Wide-Resnet-50. Since slice-level classification is the most important stage in our approach, we investigated different scenarios. The investigated scenarios include data prepossessing, data augmentation, and training deep learning architectures with the multi-tasks strategy.

\subsubsection{Stacking Grayscale Image}

Since the slice read from the '.dcm' file is a one-channel grayscale image and the input of the pretrained CNN models (on ImageNet) includes three channels (RGB images), we stacked the grayscale slice to obtain an RGB-like image. In this experiment, all CNN architectures were fully trained using transfer learning for 20 epochs with the Adam optimizer [36]. The initial learning rate is 0.0001 , which decays by 0.1 after 10 epochs, followed by another decay of 0.1 after 15 epochs.

Table 1 summarizes the obtained results. From these results, we notice that the four CNN architectures achieved similar results with slightly better results using the Densenet161 architecture. Figure 6 contains the confusion matrices of the trained CNN architectures (ResneXt-50, Densenet-161, Inception-v3, and Wide-Resnet-50) on the validation data. From these confusion matrices, we notice that the Densenet-161 architecture achieved the 
best performance in the recognition of COVID-19 and Cap slices with accuracies of $65.87 \%$ and $56.19 \%$, respectively. On the other hand, for normal slice recognition, the Inception-v3 architecture achieved the best performance with an accuracy of $93.73 \%$.

Table 1. Validation slice-level classification results using stacked grayscale images with four backbone CNN architectures (ResneXt-50, Densenet-161, Inception-v3, and Wide-Resnet-50).

\begin{tabular}{cc}
\hline Model & Slice-Level Classification (\%) \\
\hline ResneXt-50 & 80.80 \\
Densenet-161 & 81.81 \\
Inception-v3 & 81.78 \\
Wide-Resnet-50 & 80.72 \\
\hline
\end{tabular}
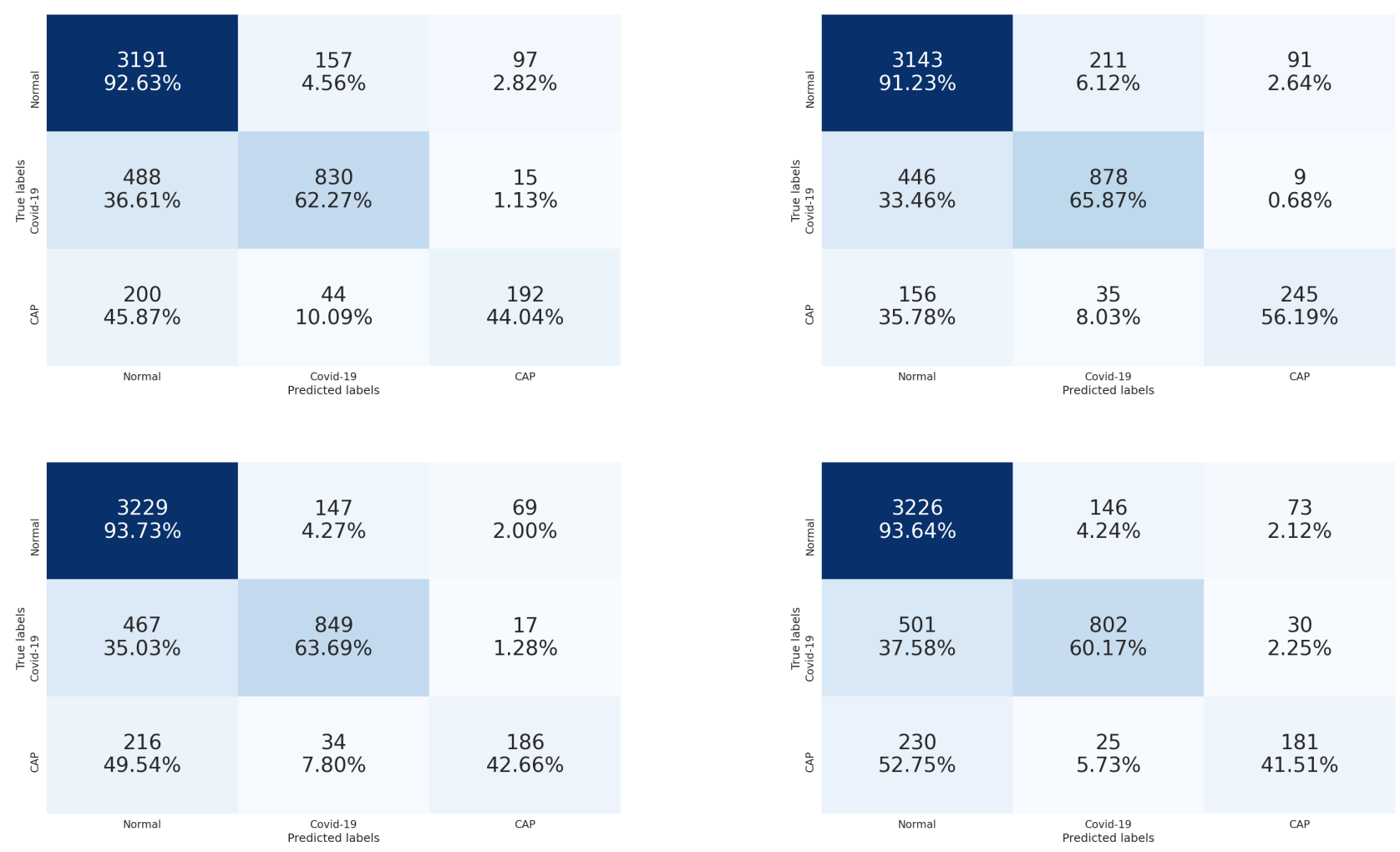

Figure 6. Confusion matrices of the stacked grayscale images on validation data using ResneXt-50, Inception-v3, DenseNet161, and Ensemble-CNNs, respectively. The vertical axis is for the true classes, and the horizontal axis is for the predicted classes.

\subsubsection{Stacking Grayscale Image with the Segmented Result}

In this experiment, we proposed segmenting the lung lobes and then stacking the segmentation result with the raw slice image to obtain an RGB-like image as illustrated in Figure 3. Similar to the stacking grayscale experiment, all CNN models were fine-tuned for 20 epochs with the Adam optimizer [36]. The initial learning rate is 0.0001, which decays by 0.1 after 10 epochs, followed by another decay of 0.1 after 15 epochs. Table 2 summarizes the obtained results of the four $\mathrm{CNN}$ architectures. From these results, we notice that the Densenet-161 architecture achieved a slightly better result than the other three $\mathrm{CNN}$ architectures. In general, the four $\mathrm{CNN}$ architectures achieved close results.

Figure 7 contains the confusion matrices of the trained CNN architectures (ResneXt50, Densenet-161, Inception-v3, and Wide-Resnet-50) on the validation data. From these confusion matrices, we notice that the Wide-Resnet- 50 architecture achieved the best performance on the recognition of normal and COVID-19 slices with accuracies of $94.78 \%$ 
and $84.47 \%$, respectively. On the other hand, for Cap slices recognition, the Densenet- 161 architecture achieved the best performance with an accuracy of $58.03 \%$.

By comparing the results of Tables 1 and 2, we notice that stacking the segmentation of lung lobes considerably improved the results of all four CNN architectures. Similarly, when we compare the confusion matrices of Figures 6 and 7, we notice that the best recognition rate of all three classes improved, especially for the COVID-19 rate, which improved by $18.6 \%$. This proves the importance of stacking the segmented lung lobes with the grayscale image in our approach.

Table 2. Validation slice-level classification results using stacked grayscale image with four backbone CNN architectures (ResneXt-50, Densenet-161, Inception-v3, and Wide-Resnet-50).

\begin{tabular}{cc}
\hline Model & Slice-Level Classification (\%) \\
\hline ResneXt-50 & 87.86 \\
Densenet-161 & 88.10 \\
Inception-v3 & 88.03 \\
Wide-Resnet-50 & 87.76 \\
\hline
\end{tabular}
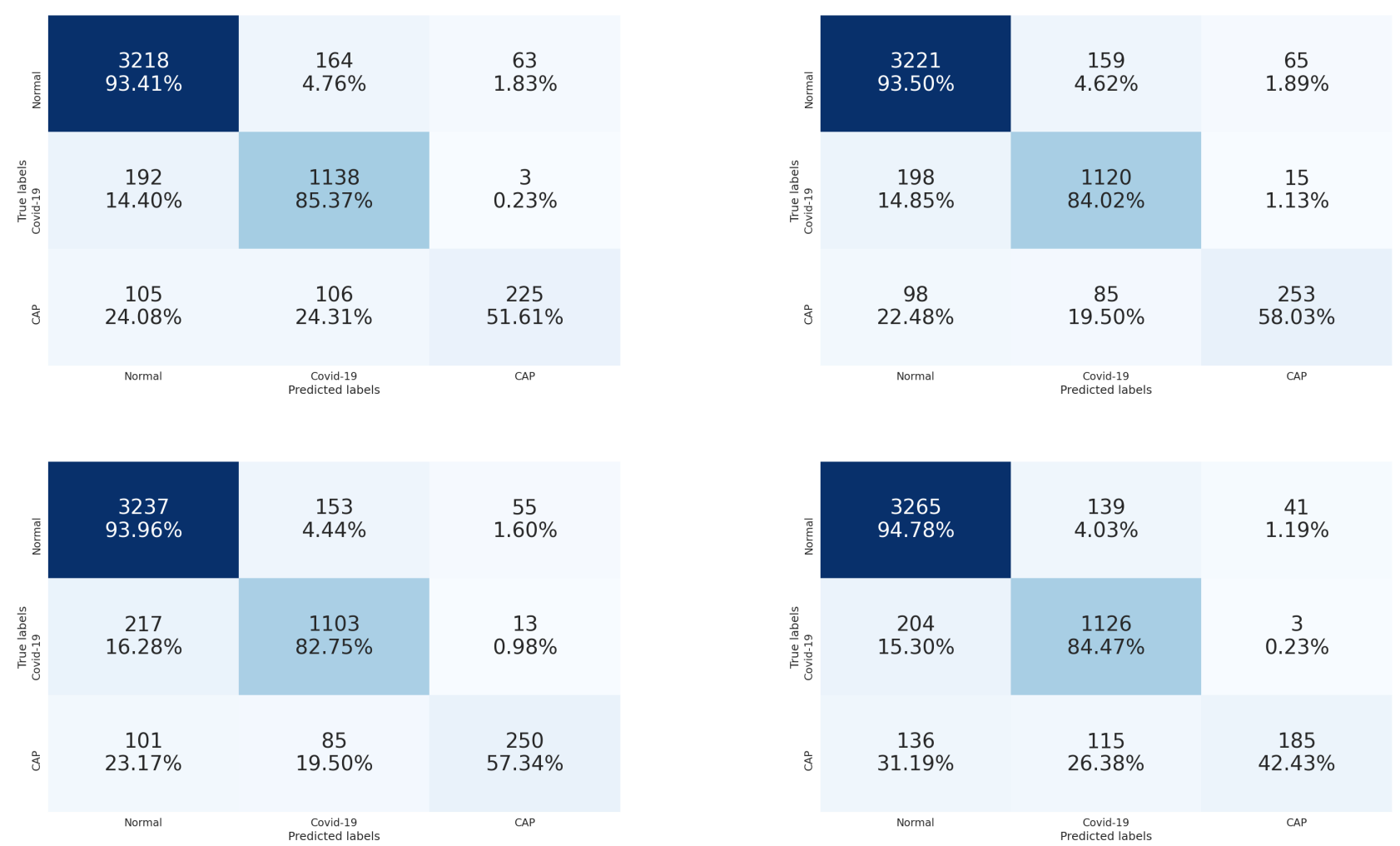

Figure 7. Confusion matrices of the stacked grayscale image and the segmented lung lobes on validation data using ResneXt-50, Densenet-161, Inception-v3, and Wide-Resnet-50, respectively. The vertical axis is for the true classes, and the horizontal axis is for the predicted classes.

\subsubsection{Data Augmentation of the Stacked Gray and Segmented Images Scenario}

In this experiment, we proposed augmenting the stacked gray and segmented lung lobes to have a balanced database as described in Section 3.3.1. Since the data are augmented in this scenario, we fine-tuned the $\mathrm{CNN}$ models for only 10 epochs with the Adam optimizer [36]. The initial learning rate is 0.0001 , which decays by 0.1 after 3 epochs, followed by another decay of 0.1 after 6 epochs. Table 3 summarizes the obtained results of four CNN architectures using the augmented data. From these results, we notice that the Inception-v3 architecture achieved a slightly better result than the other three CNN 
architectures. In comparison with the results of Table 2, we notice that the performance of the four CNN architectures improved.

Figure 8 contains the confusion matrices of the trained CNN architectures (ResneXt-50, Densenet-161, Inception-v3, and Wide-Resnet-50) on the validation data. From these confusion matrices, we notice that the ResneXt-50, Inception-v3, and Densenet-161 architectures achieved the best performance on the recognition of normal (94.43\%), COVID-19 (84.32\%), and Cap slices $(68.81 \%)$, respectively. In this scenario, we observe that the recognition rate of the Cap slices has improved by about $10 \%$ compared with the previous scenario. This proves the efficiency of using data augmentation techniques.

Table 3. Validation slice-level classification results using augmented stacked gray and segmented images scenario with four backbone CNN architectures (ResneXt-50, Densenet-161, Inception-v3, and Wide-Resnet-50).

\begin{tabular}{cc}
\hline Model & Slice-Level Classification (\%) \\
\hline ResneXt-50 & 88.51 \\
Densenet-161 & 88.42 \\
Inception-v3 & 88.64 \\
Wide-Resnet-50 & 87.97 \\
\hline
\end{tabular}
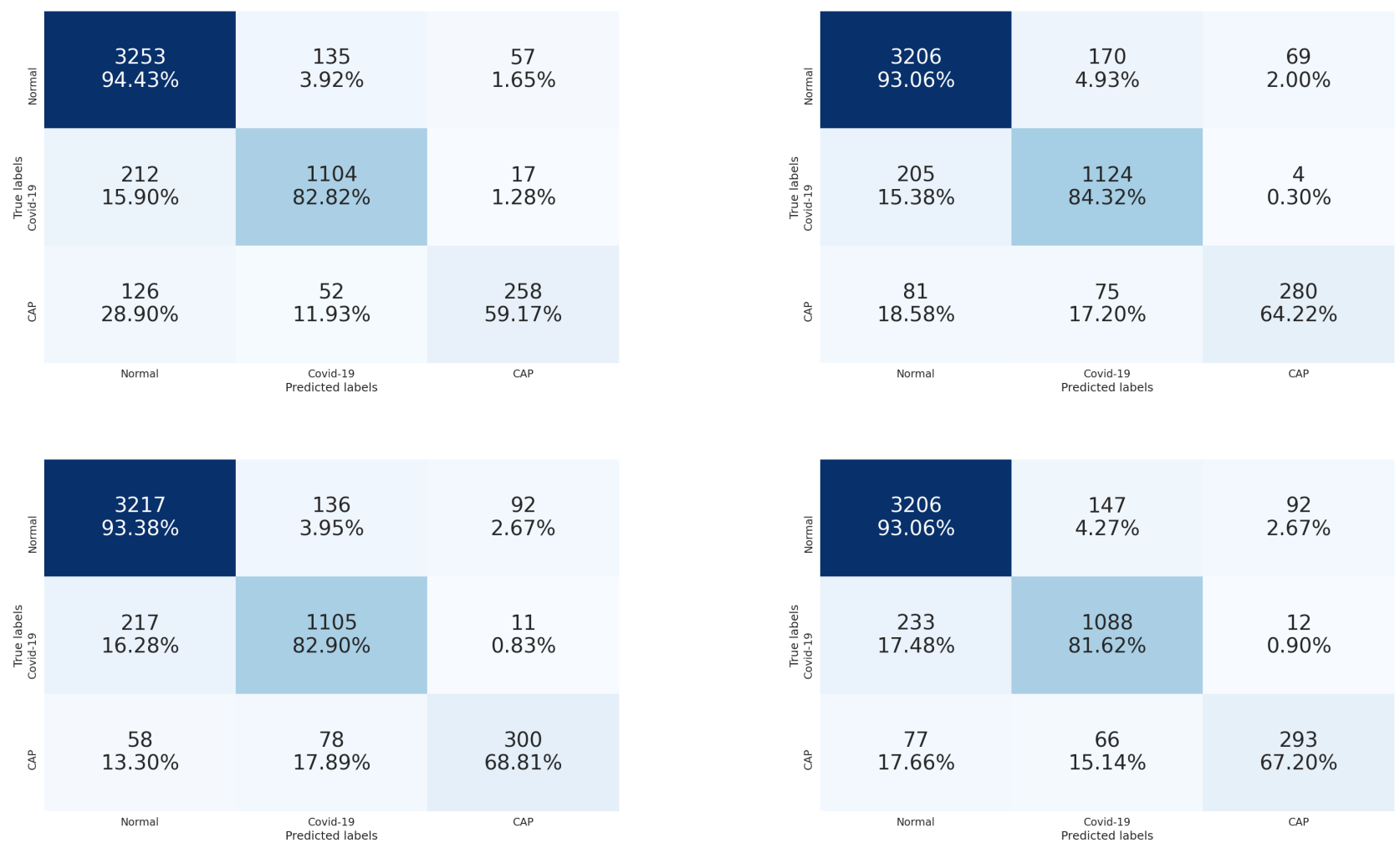

Figure 8. Confusion matrices of the augmented stacked grayscale image and the segmented lung lobes on validation data using ResneXt-50, Densenet-161, Inception-v3, and Wide-Resnet-50, respectively. The vertical axis is for the true classes, and the horizontal axis is for the predicted classes.

\subsubsection{Multi-Tasks Experiment}

In this experiment, we trained the augmented data of the stacked gray and segmented scenario using the multi-tasks paradigm as explained in Section 3.3.1. The first task is to classify the input stacked image (gray and segmented) into normal, COVID-19, and Cap classes. Since the size and the shape of the lungs change from the first, middle, and last slices of the CT scan, we divided each CT scan into five equalized regions. The slices within 
the same region in the CT scan will have the same label. In addition to the main task (to classify the slices into normal, COVID-19, and Cap), the second task classifies the slice into one of the region classes. For the same purpose, we added the total number of slices of the CT scan and the slice location in the CT scan into the deep features (FC layer) as shown in Figure 4. The aim of the second task of the multi-tasks is to make the model aware of the morphological changes of the slice order in the CT scan. Both tasks use the focal loss function [35] with gamma equal to 1.5. The overall loss is the sum of the two tasks' loss with weights of 1 and 0.3 for the first and secondary task, respectively, thus giving more importance to the main classification task (classifying the slice into normal, COVID-19, or Cap).

Since the augmented data are used in this scenario, we fine-tuned the CNN models for only 10 epochs with the Adam optimizer [36]. The initial learning rate is 0.0001 , which decays by 0.1 after 3 epochs, followed by another decay of 0.1 after 6 epochs. The obtained results are summarized in Table 4 . From these results, we notice that the architectures achieved a close result on classifying the slices with slightly better performance by ResneXt50 architecture. Compared with the results of the previous scenario (Table 3), we notice that the performance improved for all $\mathrm{CNN}$ architectures.

Figure 9 contains the confusion matrices of the trained CNN architectures (ResneXt-50, Densenet-161, Inception-v3, and Wide-Resnet-50) on the validation data. From these confusion matrices, we notice that Wide-Resnet-50, Densenet-161, and ResneXt-50 architectures achieved the best performance on the recognition of normal (94.19\%), COVID-19 (83.80\%), and Cap slices $(70.64 \%)$, respectively. In this scenario, we observe that the best recognition rate of the Cap slices has improved. Subsequently, the multi-tasks strategy is efficient for the hardest class recognition.
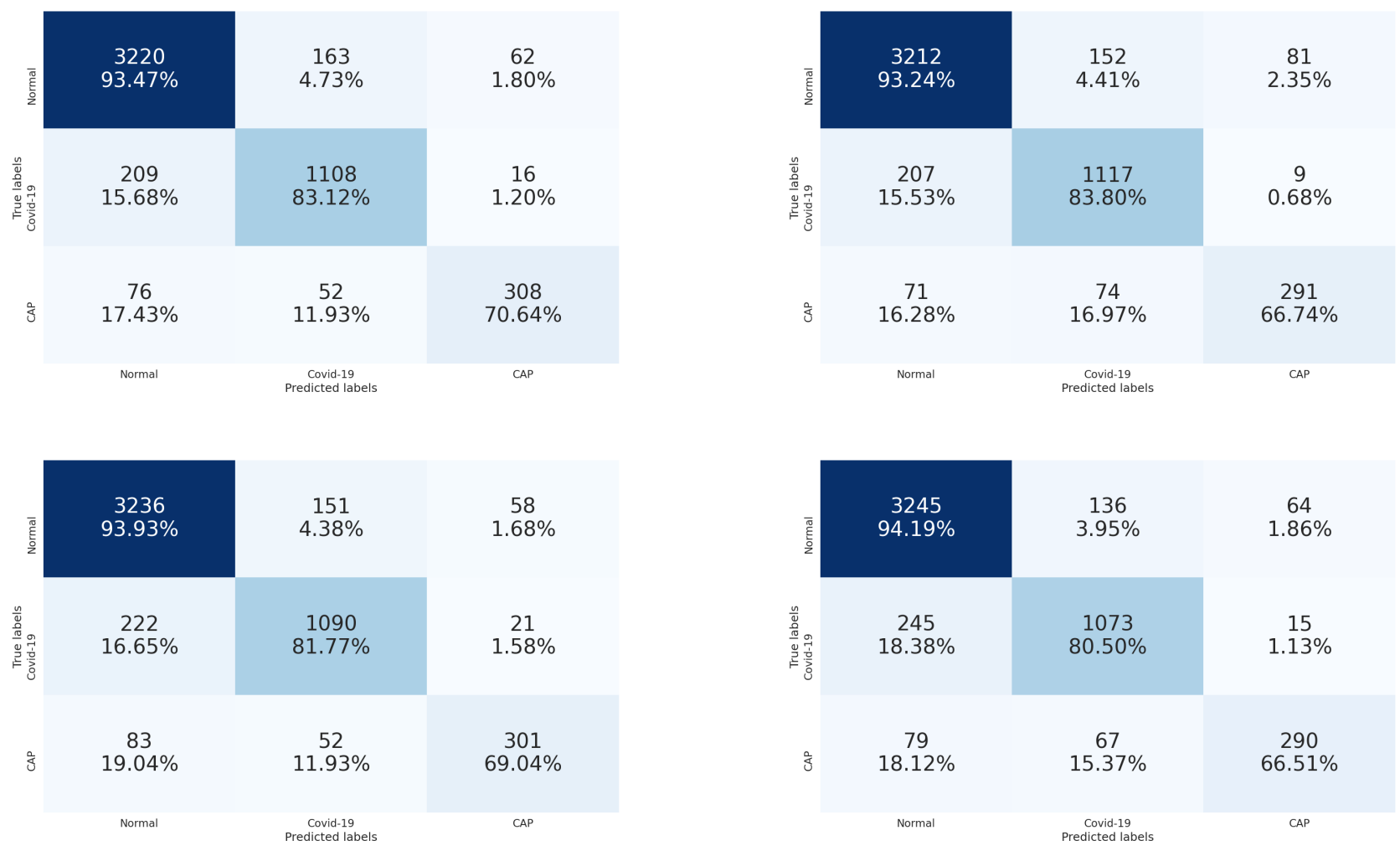

Figure 9. Confusion matrices of the three classes of COVID-19 testing data using ResneXt-50, Densenet-161, Inception-v3, and Wide-Resnet-50, respectively. The vertical axis is for the true classes, and the horizontal axis is for the predicted classes. 
Table 4. Validation of slice-level classification results using multi-tasks learning with four backbone CNN architectures (ResneXt-50, Densenet-161, Inception-v3, and Wide-Resnet-50) [6].

\begin{tabular}{cc}
\hline Model & Slice-Level Classification (\%) \\
\hline ResneXt-50 & 88.91 \\
Densenet-161 & 88.61 \\
Inception-v3 & 88.74 \\
Wide-Resnet-50 & 88.37 \\
\hline
\end{tabular}

\subsubsection{Patient-Level Classification}

In this section, we used the trained CNN architectures (ResneXt-50, Densenet-161, Inception-v3, and Wide-Resnet-50) using the multi-tasks strategy for slice-level classification. Then, we used the prediction percentage of the three classes within the CT scan regions (each CT scan is divided into 20 regions) to train and test two classifiers, which are SVM and XG-boost, for CT scan classification. The obtained results on the validation data are summarized in Table 5. From these results, we notice that the Inception-v3 features with the XG-boost classifier achieved the best performance compared with the other architectures' features. On the other hand, combining all four models' features with the XG-boost classifier achieved the best performance. In contrast, combining features with the SVM classifier achieved the same result as the Wide-Resnet- 50 features. This proves the efficiency of using the XG-boost classifier for single architecture features as well as combining all architectures' features.

Table 5. Validation CT scan classification results using SVM and XG-boost with different grouping percentages' features (ResneXt-50, Densenet-161, Inception-v3, and Wide-Resnet-50 grouping percentages features) and their combination [6].

\begin{tabular}{ccc}
\hline \multirow{2}{*}{ Model } & \multicolumn{2}{c}{ CT Scans Classification (\%) } \\
\cline { 2 - 3 } & SVM & XG-Boost \\
\hline ResneXt-50 features & 81.63 & 79.59 \\
Densenet-161 features & 82.65 & 82.65 \\
Inception-v3 features & 82.65 & 85.71 \\
Wide-Resnet-50 features & 80.61 & 80.61 \\
\hline CNR-IEMN & 80.61 & 87.75 \\
\hline
\end{tabular}

From the confusion matrix in Figure 10, we notice that our approach achieved high accuracy in the recognition of normal and COVID-19 CT scans, where it achieved 95.83\% and $96.36 \%$, respectively. On the other hand, our approach achieved $52.63 \%$ for the recognition of Cap CT scans, and this because the Cap class has less training data for both slice-level and patient-level stages than the normal and COVID-19 classes.

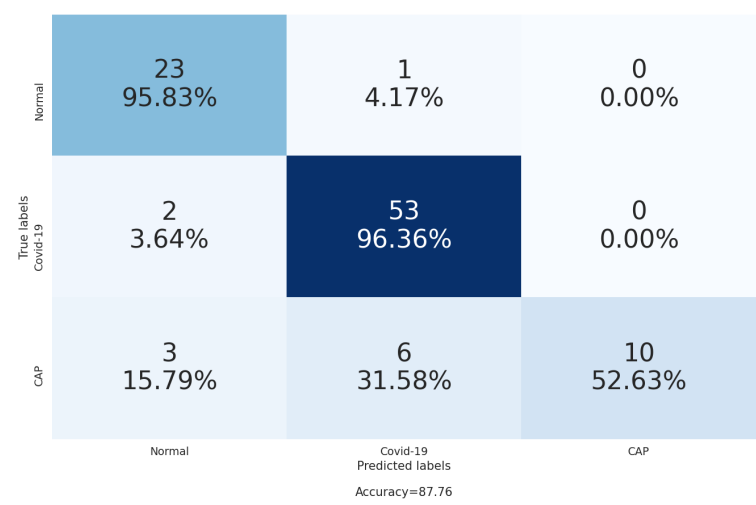

Figure 10. The confusion matrix of the CT scans classification on the validation data [6]. 


\subsection{Testing Data}

In the SPGC on COVID-19 challenge, there are 17 participant teams. The testing data labels are not known by all participating teams. The overall results and rankings of the 17 teams are illustrated in Figure 11. From these results, our approach achieved fifth place in the SPGC on COVID-19 challenge. In more detail, our approach achieved $81.11 \%$ as overall accuracy and $91.43 \%, 45.0 \%$, and $91.43 \%$ sensitivities for COVID-19, Cap, and normal, respectively. Compared with other participants, our approach achieved the best sensitivity for the COVID-19 class.

$\begin{array}{lrrrr}\text { Team } & \text { Accuracy } & \text { COVID-19 Sensitivity } & \text { CAP Sensitivity } & \text { Normal Sensitivity } \\ \text { TheSaviours } & 0.9000 & 0.8571 & 0.9000 & 0.9429 \\ \text { IITDelhi } & 0.8889 & 0.8857 & 0.9000 & 0.8857 \\ \text { LLSCP } & 0.8778 & 0.8000 & 0.9000 & 1.0000 \\ \text { UniSheff_EEE } & 0.8556 & 0.8286 & 0.8000 & 0.9143 \\ \text { CNRIEMEN } & 0.8111 & 0.9143 & 0.4500 & 0.9143 \\ \text { FushionSTAR } & 0.8000 & 0.9143 & 0.3000 & 0.9714 \\ \text { Bingo } & 0.8000 & 0.8857 & 0.3500 & 0.9714 \\ \text { SAIVT } & 0.8000 & 0.6857 & 0.9500 & 0.8286 \\ \text { Winter } & 0.7667 & 0.8571 & 0.7000 & 0.7143 \\ \text { Ewen } & 0.7111 & 0.8000 & 0.7000 & 0.6286 \\ \text { PRIME } & 0.7111 & 0.6000 & 1.0000 & 0.6571 \\ \text { Deepcam } & 0.6778 & 0.8857 & 1.0000 & 0.2857 \\ \text { THN } & 0.6667 & 0.6000 & 0.9500 & 0.5714 \\ \text { CVLAB } & 0.4556 & 0.8000 & 0.4000 & 0.1429 \\ \text { NRS } & 0.4222 & 0.3714 & 0.1000 & 0.6571 \\ \text { Sheerin } & 0.4000 & 0.8857 & 0.1000 & 0.0857 \\ \text { SSU } & 0.3444 & 0.3714 & 0.7000 & 0.4000\end{array}$

Figure 11. The final results of the three test sets.

The results of the first, second, and third testing splits are summarized in Tables 6-8, respectively. From these tables, we notice that our approach achieved second place in the first and second testing splits. On the other hand, our approach achieved 10th place in the third testing split. Despite our rank in this testing split, our approach recognized the COVID-19 and normal cases well. In this testing split, most of the CT scans have a history of heart disease or operation with an abnormal manifestation related to a non-infection disease. This highly influenced our recognition approach for CAP cases.

Table 6. First testing split results.

\begin{tabular}{ccccc}
\hline Team & COVID-19 & Cap & Normal & Total \\
\hline TheSaviours & $9 / 10$ & $8 / 10$ & $9 / 10$ & $26 / 30$ \\
CNR-IEMN & $8 / 10$ & $7 / 10$ & $9 / 10$ & $24 / 30$ \\
IITDelhi & $9 / 10$ & $8 / 10$ & $6 / 10$ & $23 / 30$ \\
UniSheff_EEE & $9 / 10$ & $7 / 10$ & $7 / 10$ & $23 / 30$ \\
Bingo & $10 / 10$ & $0 / 10$ & $10 / 10$ & $20 / 30$ \\
FushionSTAR & $10 / 10$ & $0 / 10$ & $10 / 10$ & $20 / 30$ \\
\hline
\end{tabular}


Table 7. Second testing split results.

\begin{tabular}{cccc}
\hline Team & COVID-19 & Normal & Total \\
\hline LLSCP & $15 / 15$ & $15 / 15$ & $30 / 30$ \\
CNR-IEMN & $14 / 15$ & $14 / 15$ & $28 / 30$ \\
FushionSTAR & $14 / 15$ & $14 / 15$ & $28 / 30$ \\
UniSheff_EEE & $13 / 15$ & $15 / 15$ & $28 / 30$ \\
SAIVT & $13 / 15$ & $14 / 15$ & $27 / 30$ \\
Bingo & $12 / 15$ & $15 / 15$ & $27 / 30$ \\
\hline
\end{tabular}

Table 8. Third testing split results.

\begin{tabular}{ccccc}
\hline Team & COVID-19 & Cap & Normal & Total \\
\hline IITDelhi & $10 / 10$ & $10 / 10$ & $10 / 10$ & $30 / 30$ \\
LLSCP & $9 / 10$ & $10 / 10$ & $10 / 10$ & $29 / 30$ \\
TheSaviours & $9 / 10$ & $9 / 10$ & $9 / 10$ & $29 / 30$ \\
Deepcam & $10 / 10$ & $10 / 10$ & $8 / 10$ & $28 / 30$ \\
Ewen & $10 / 10$ & $9 / 10$ & $8 / 10$ & $27 / 30$ \\
.. &. &.. &.. &. \\
CNR-IEMN & $10 / 10$ & $2 / 10$ & $9 / 10$ & $21 / 30$ \\
\hline
\end{tabular}

\subsection{Segmentation Influence}

Stacking segmented lung lobes with the grayscale image proved its efficiency (by comparing the results of Tables 1 and 2). Despite this considerable improvement, the recognition of Cap slices needs more improvement. Figure 12 shows some segmented slices for COVID-19 and Cap cases. From Figure 12, we notice that the segmented results considered infection parts as lung lobes especially for Cap slices (row 2 and 3 Figure 12). One possible way to improve the performance of our approach is to use a CNN-based approach that were trained on infected slices to segment the lung lobes.
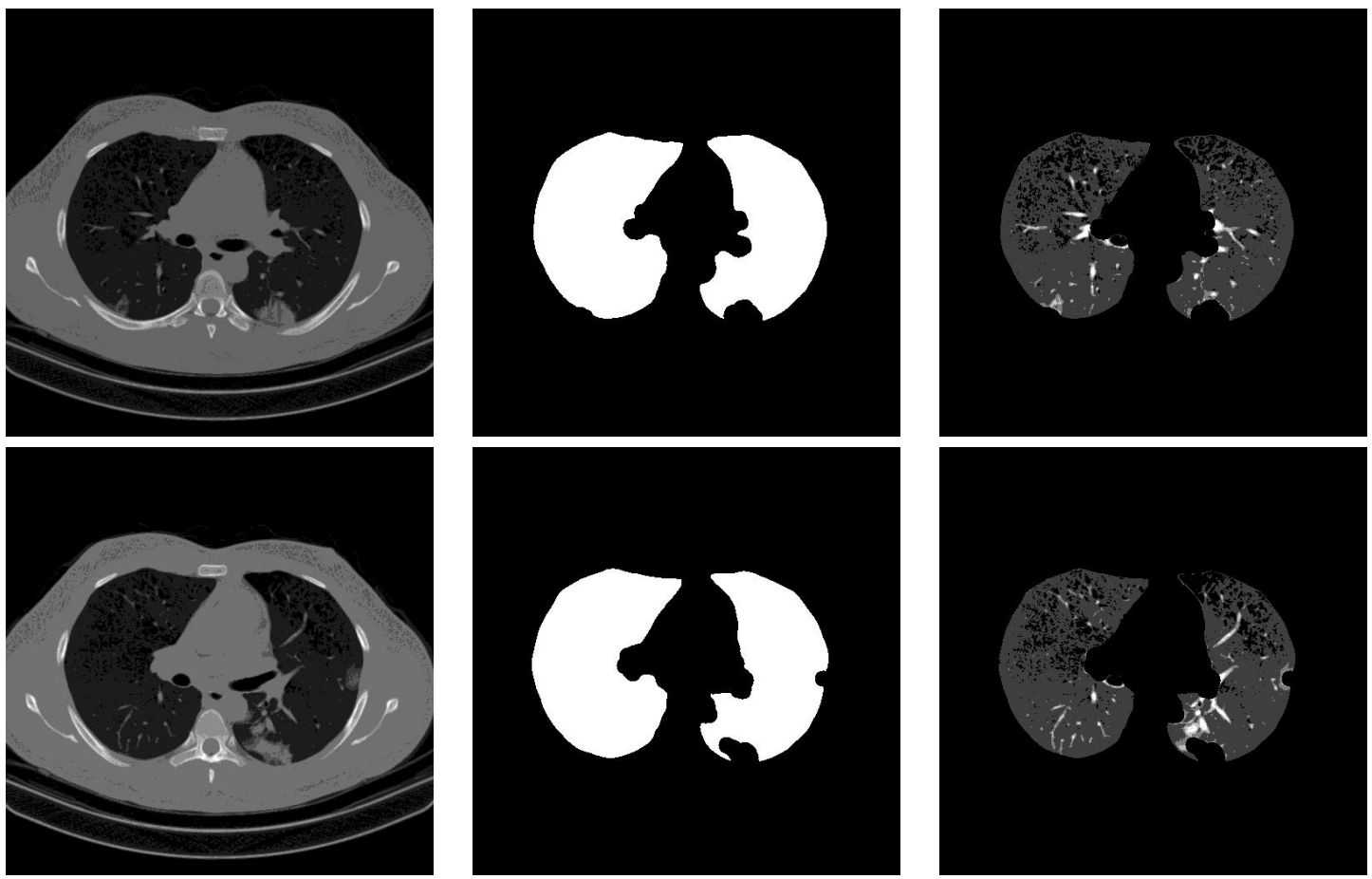

Figure 12. Cont. 

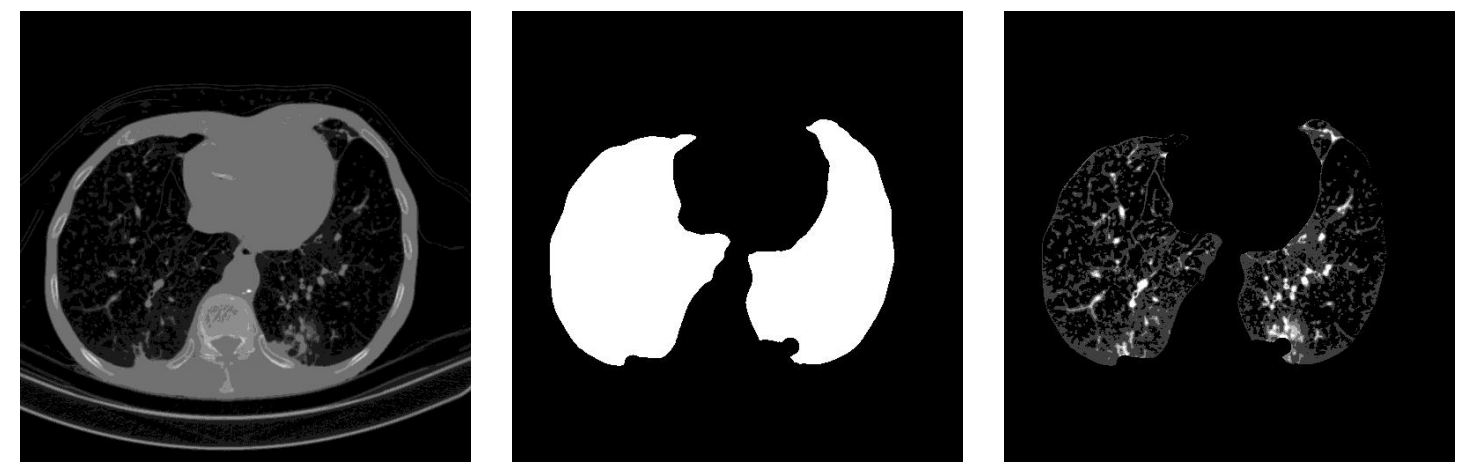

Figure 12. Segmented lung lobes of infected slices with COVID-19 and Cap. Rows 1, 2, and 3 are for COVID-19, Cap, and Cap slices, respectively.

\subsection{Heat Map}

To have more intuition about the most important lung regions that the CNN architectures consider to classify the slice images, we used the randomized input sampling for explanations (RISE) approach [37]. Figures 13-15 consist of two heat map examples of COVID-19, Cap, and normal cases, respectively. In the first example from Figure 13, we notice that despite the lung segmentation being not fully correct (it misses a considerable part of the infected region), the four CNN architectures gave more importance to the infected regions and to the lung regions in general. In the second example from Figure 13, we observe that the lung lobes were precisely segmented, and the heat maps of the four CNN architectures were well defined the infected regions, especially the Wide-Resnet-50 architecture. From both Cap examples in Figure 14, we notice that despite the lung segmentation missing considerable infected parts as well as the lung lobe regions, the heat maps of all CNN architectures give more importance to the infected region and to the lung lobes in general. This proves that the trained CNN architecture is able to define the regions of interest even when the lung lobes segmentation is not good. For the normal slice examples in Figure 15, the trained CNN architectures gave more importance to the lung lobes, especially the lower region, since the infection usually occurs there. The heat maps of COVID-19, Cap, and normal cases prove that the CNN architectures learned precisely where to look to identify the infection from the slice images.
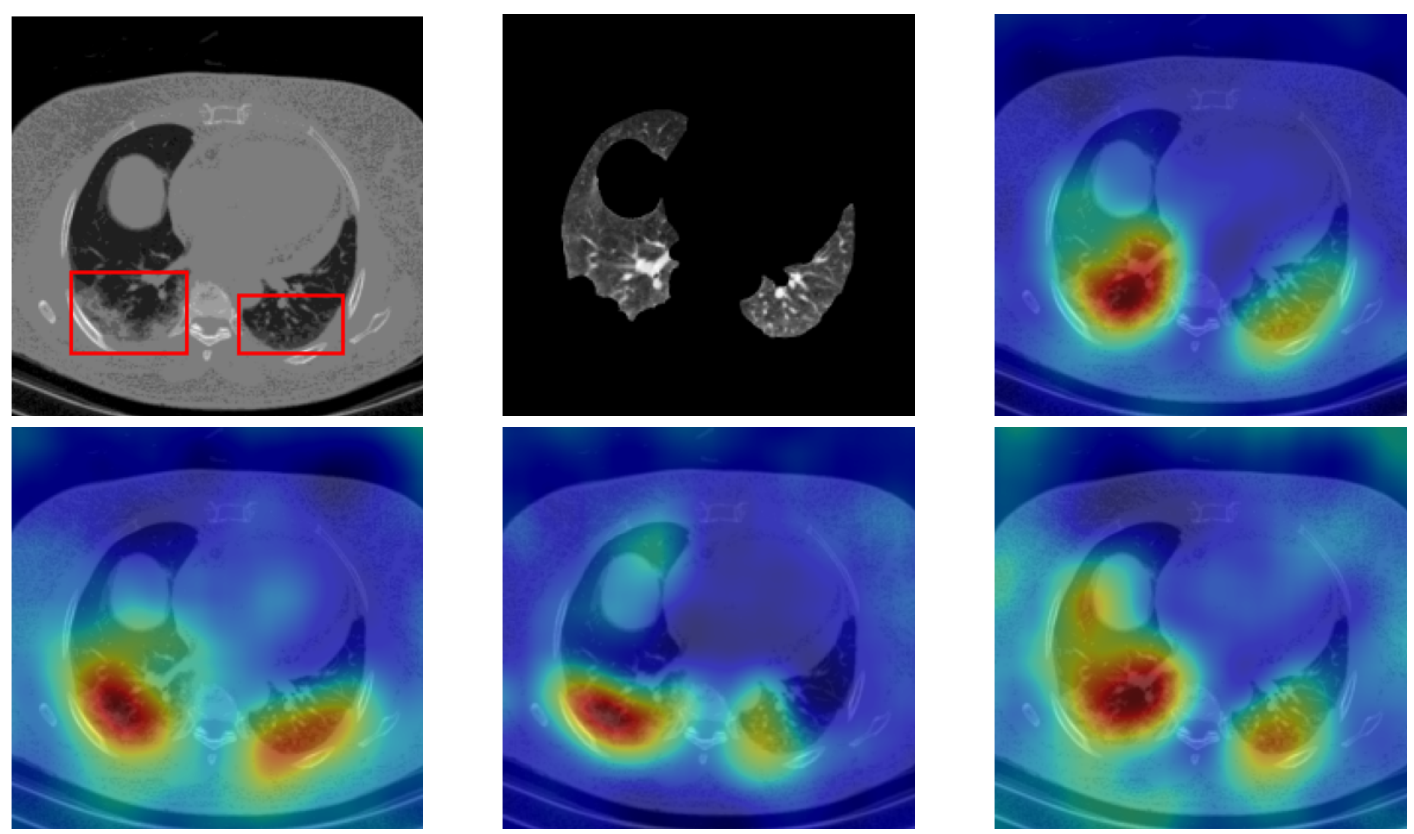

Figure 13. Cont. 

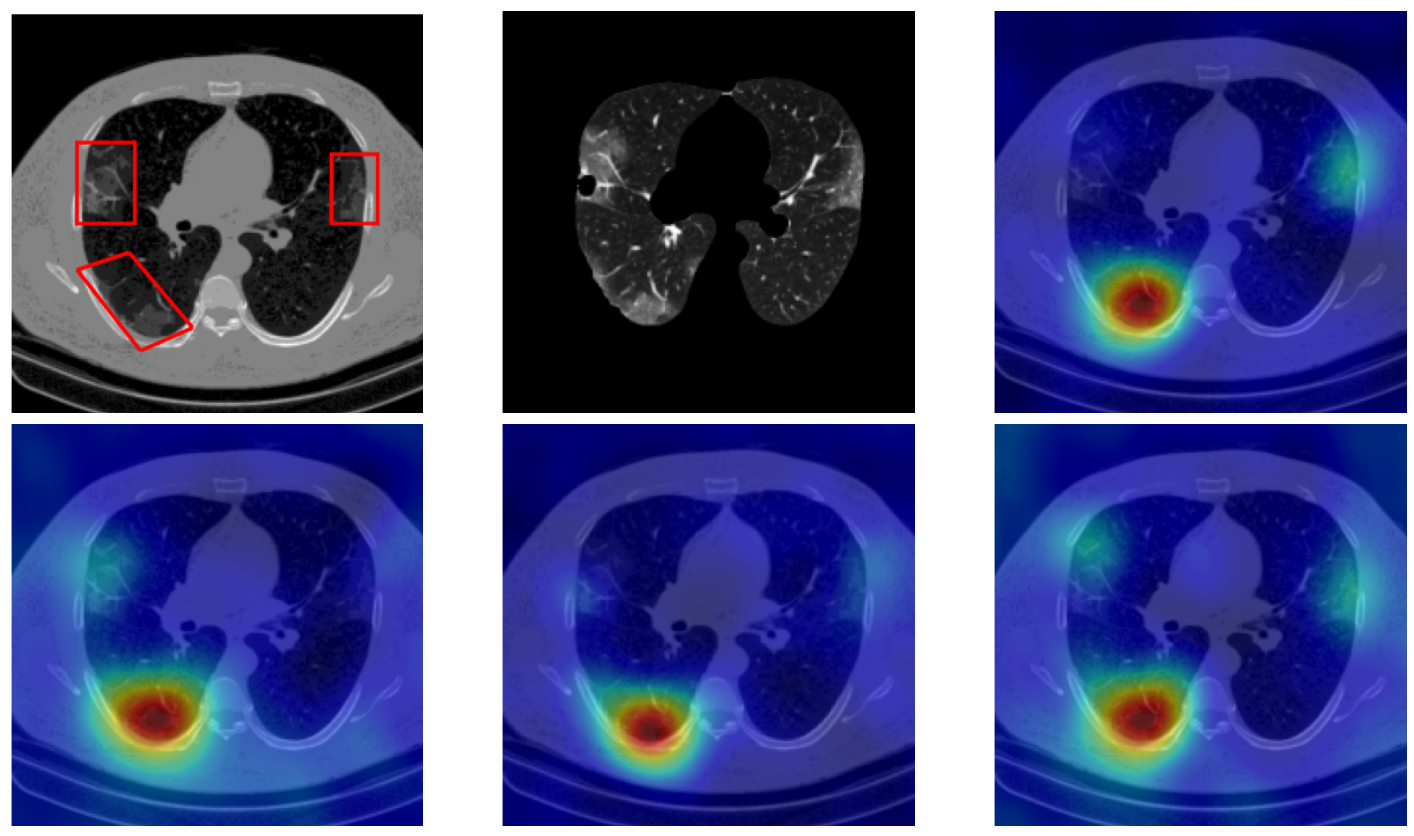

Figure 13. RISE heat map examples of COVID-19 slice images using the trained multi-tasks CNN architectures (ResneXt-50, Densenet-161, Inception-v3, and Wide-Resnet-50). The first example is shown in the first two rows, where the images represent the input slice image and segmented lung lobes results, followed by the heat maps of ResneXt-50, Densenet-161, Inception-v3, and Wide-Resnet-50, respectively. The second example is in rows 3 and 4.
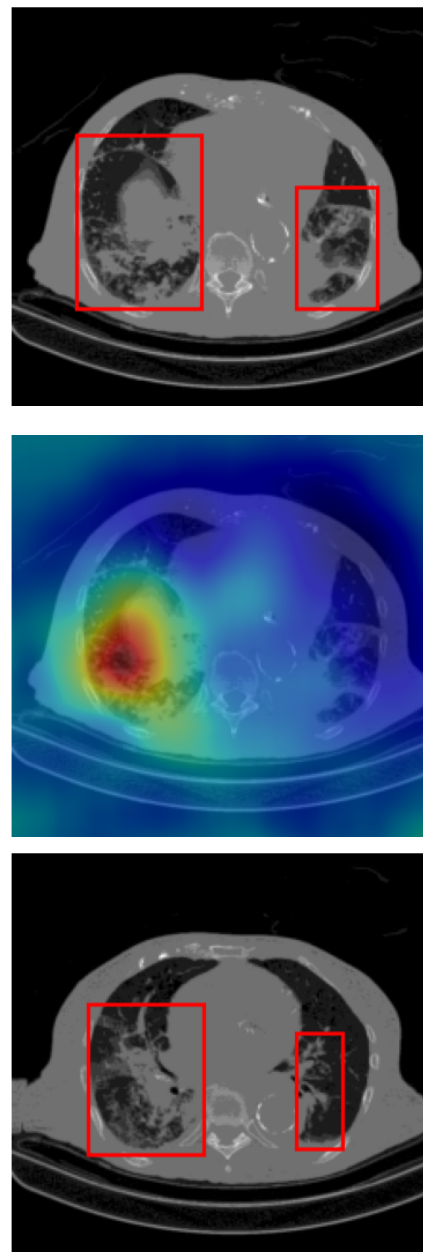
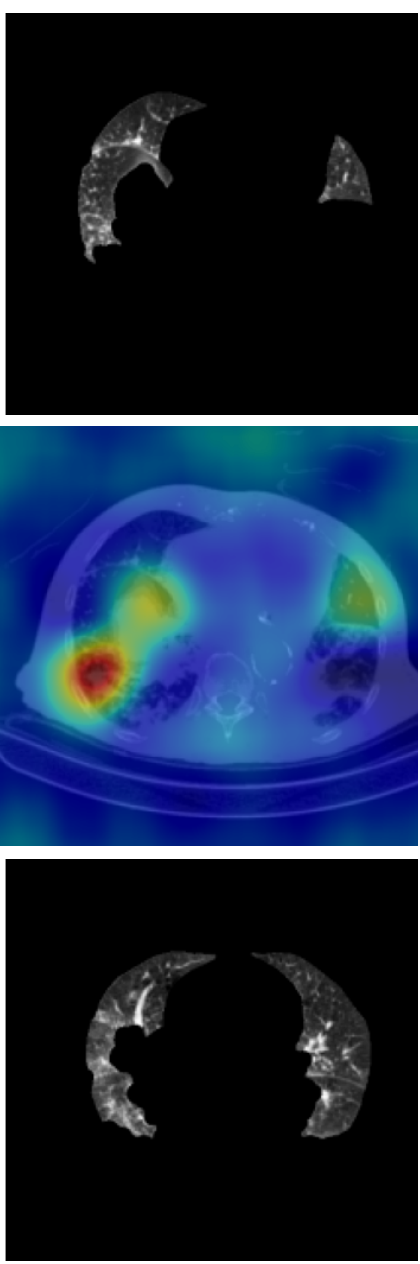

Figure 14. Cont.
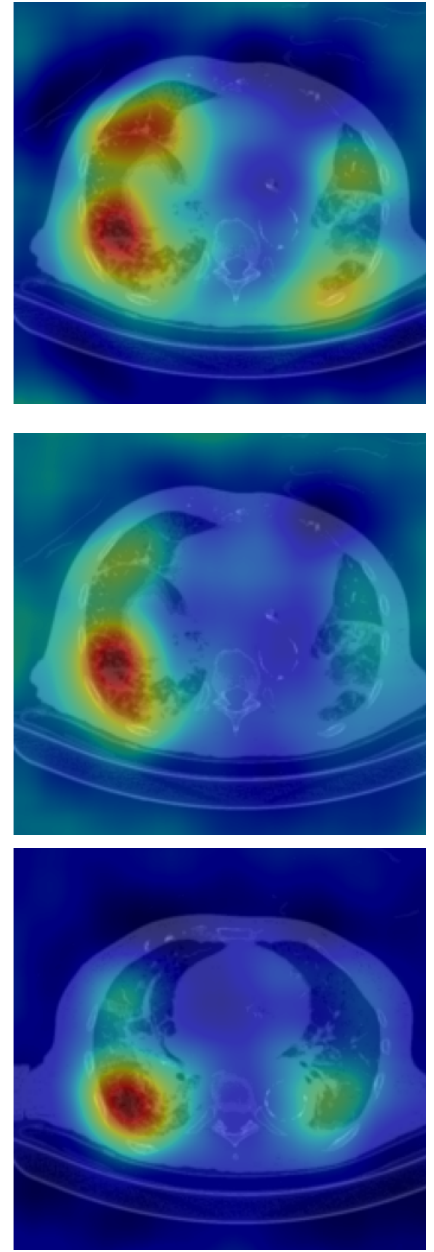

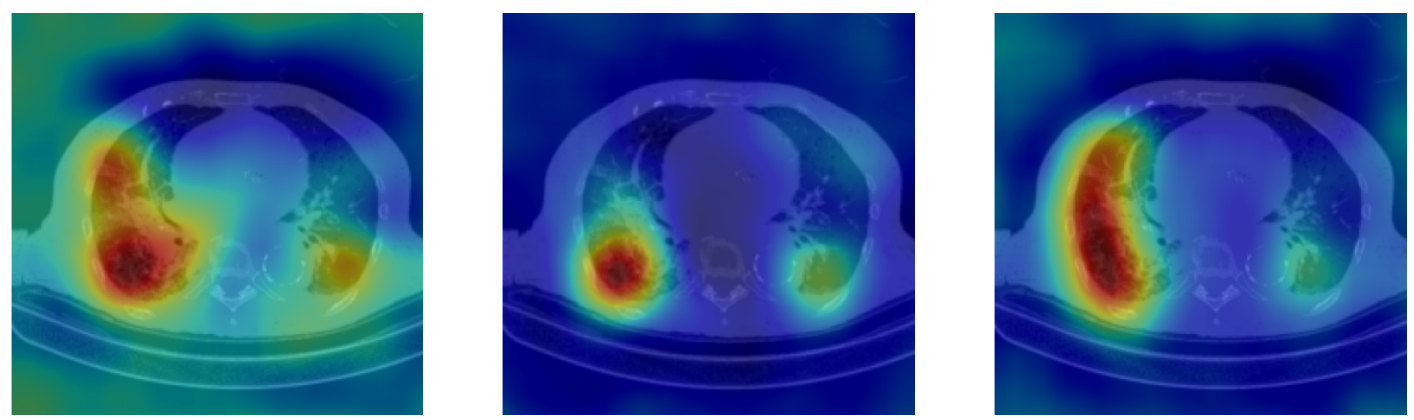

Figure 14. RISE heat map examples of Cap slice image using the trained multi-tasks CNN architectures (ResneXt-50, Densenet-161, Inception-v3, and Wide-Resnet-50). The first example is shown in the first two rows, where the images represent the input slice image and segmented lung lobes results, followed by the heat maps of ResneXt-50, Densenet-161, Inception-v3, and Wide-Resnet-50, respectively. The second example is in rows 3 and 4.
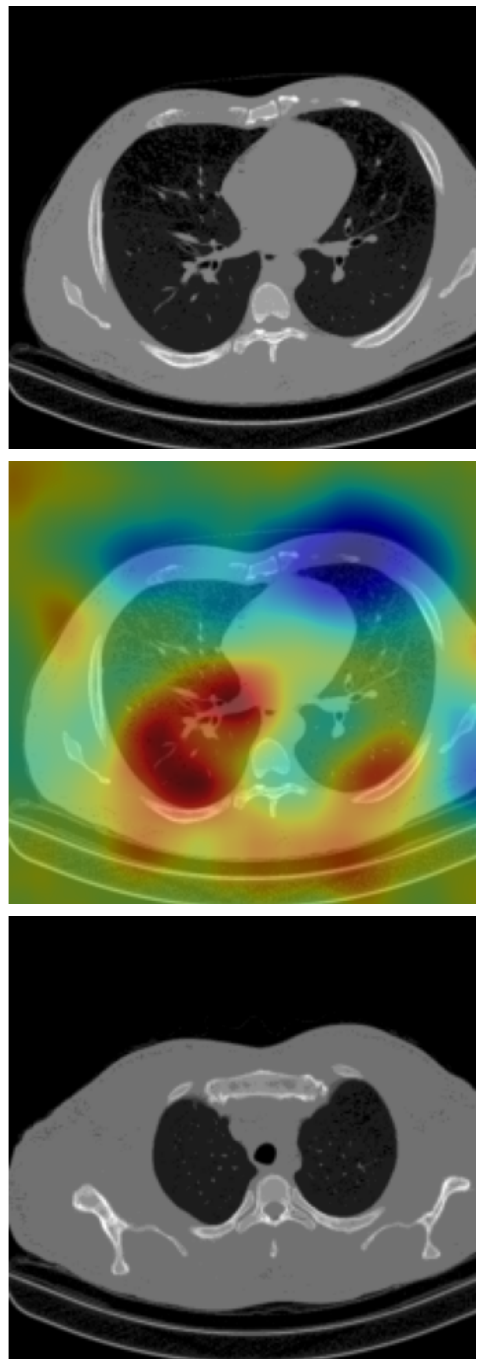
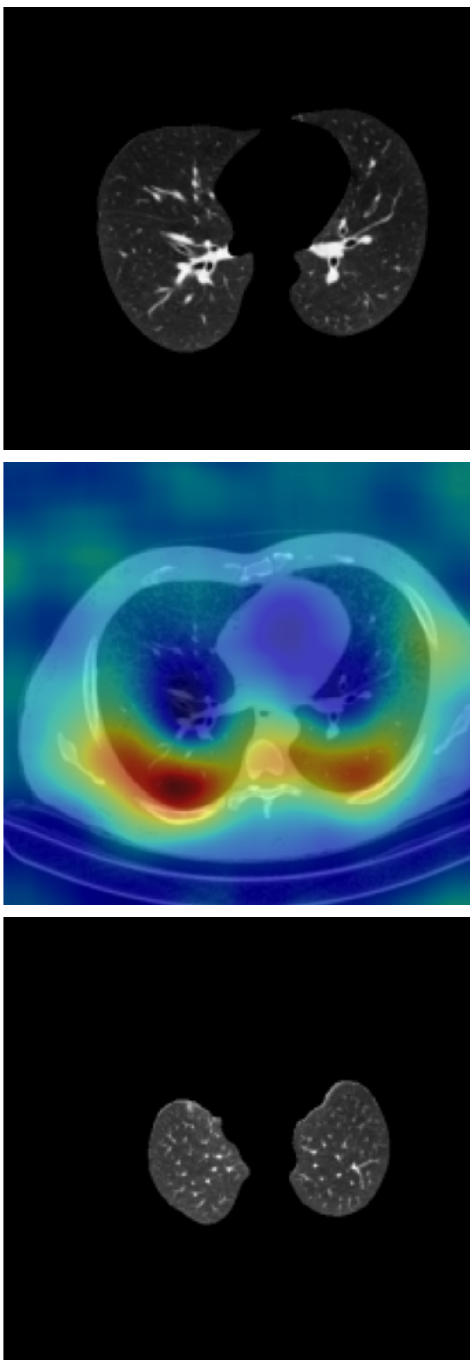

Figure 15. Cont.
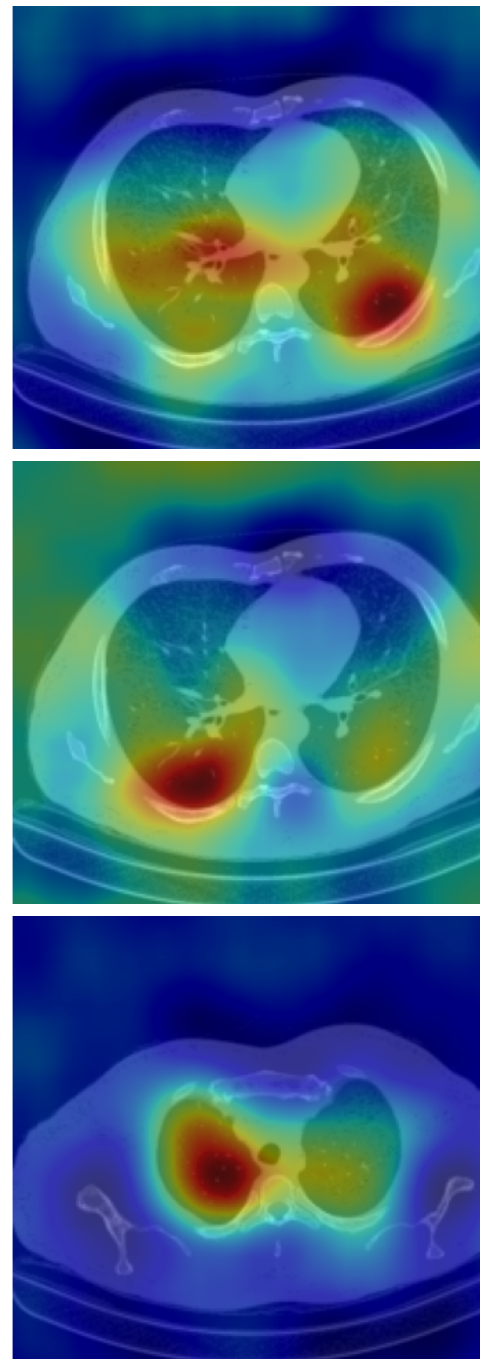

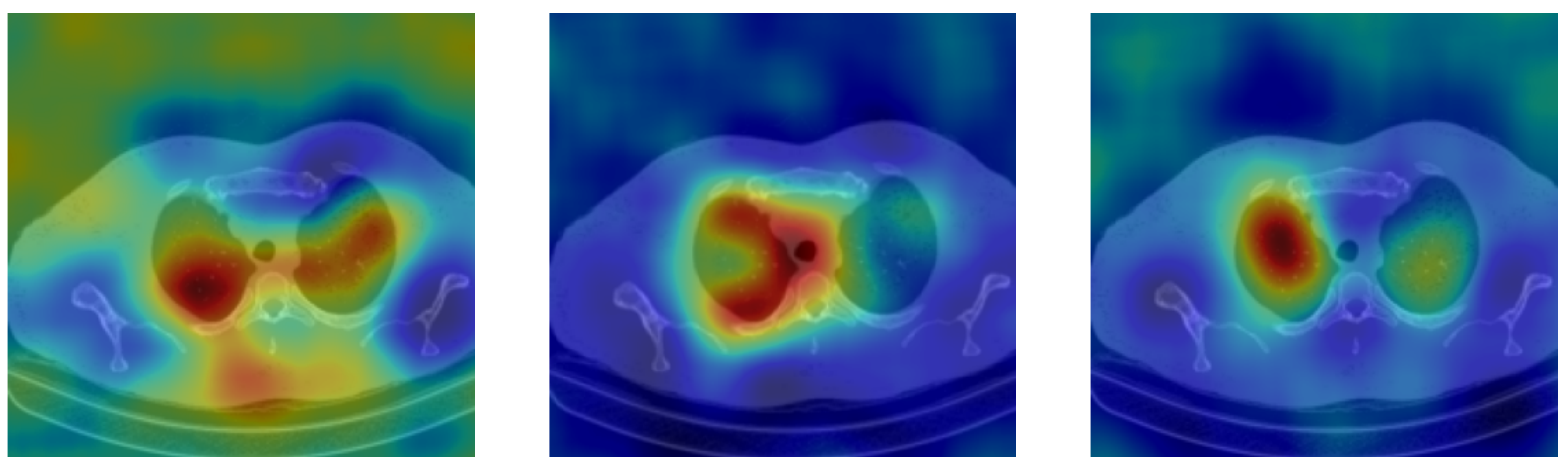

Figure 15. RISE heat map examples of normal slice image using the trained multi-tasks CNN architectures (ResneXt-50, Densenet-161, Inception-v3, and Wide-Resnet-50). The first example is shown in the first two rows, where the images represent the input slice image and segmented lung lobes results, followed by the heat maps of ResneXt-50, Densenet-161, Inception-v3, and Wide-Resnet-50, respectively. The second example is in rows 3 and 4.

\section{Conclusions}

In this paper, we presented our proposed approach (CNR-IEMN) for the 2021 COVID19 SPGC challenge, which aims to classify volumetric CT scans into normal, COVID-19, or Cap classes. To deal with the variation of the CT scans' number of slices, we proposed a two-stage deep-learning-based approach: slice-level and patient-Level classification. In the slice-level classification stage, the slice images are classified into one of three classes (normal, COVID-19, or Cap). To this end, we trained four CNN architectures (ResneXt-50, Densenet-161, Inception-v3, and Wide-Resnet-50) using multi-tasks and data augmentation strategies. As input to these CNN architectures, we stacked the grayscale slice image with its lung lobe segmentation. In the second stage, we used the trained $\mathrm{CNN}$ architectures to extract features for the whole CT scan to train the XG-boost model. The extracted features are the percentages of the predicted classes within the CT scan regions.

To evaluate the performance of our approach, we used 2021 COVID-19 SPGC challenge validation data for both slice-level and patient-level evaluations. In addition, the three test sets of the 2021 COVID-19 SPGC challenge were used for patient-level evaluation. Our approach achieved $88.91 \%$ and $87.75 \%$ as overall accuracies on the validation data for slice-level and patient-level classification, respectively. The heat maps showed that the four trained CNN models precisely located the infection in both COVID-19 and Cap slices. On the other hand, our approach achieved fifth place on the three test datasets of SPGC in the COVID-19 challenge, where our approach achieved the best result for COVID-19 sensitivity. In more detail, the overall accuracy is $81.11 \%$ with sensitivities of $91.43 \%, 45 \%$, and $91.43 \%$ for normal, COVID-19, and Cap, respectively. In addition, our approach achieved second place on two of the three testing sets.

To improve the results, especially for the Cap class, we suggest using more Cap CT scans for the slice-level and patient-level classification. Another solution to improve the performance of our approach is to use a CNN-based approach trained on infected slices to segment the lung lobes.

Author Contributions: F.B.: Data curation, Formal analysis, Investigation, Methodology, Resources, Software, Supervision, Validation, Visualization, and Writing - original draft. R.C.: Data curation, Formal analysis, Investigation, Software, Validation, Visualization, and Writing-original draft. C.D.: Data curation, Formal analysis, Funding acquisition, Investigation, Methodology, Project administration, Resources, Supervision, and Writing—original draft. A.T.-A.: Data curation, Formal analysis, Funding acquisition, Investigation, Methodology, Project administration, Resources, Supervision, and Writing - original draft. All authors have read and agreed to the published version of the manuscript.

Funding: This research received no external funding.

Institutional Review Board Statement: Not applicable. 
Informed Consent Statement: Not applicable.

Data Availability Statement: The used database were provide by the organizers of the 2021 COVID19 SPGC challenge.

Acknowledgments: The authors would like to thank Arturo Argentieri from CNR-ISASI Italy for his support with the multi-GPU computing facilities.

Conflicts of Interest: The authors declare no conflict of interest.

\author{
Abbreviations \\ The following abbreviations are used in this manuscript: \\ CNN Convolutional Neural Network \\ RT-PCR Reverse Transcription Polymerase Chain Reaction \\ SVM Support Vector Machine
}

\title{
References
}

1. Gavriatopoulou, M.; Korompoki, E.; Fotiou, D.; Ntanasis-Stathopoulos, I.; Psaltopoulou, T.; Kastritis, E.; Terpos, E.; Dimopoulos, M.A. Organ-specific manifestations of COVID-19 infection. Clin. Exp. Med. 2020, 20, 493-506. [CrossRef] [PubMed]

2. World Health Organization. Available online: https://www.who.int/emergencies/diseases/novel-coronavirus-2019 (accessed on 15 June 2021).

3. Vantaggiato, E.; Paladini, E.; Bougourzi, F.; Distante, C.; Hadid, A.; Taleb-Ahmed, A. COVID-19 Recognition Using EnsembleCNNs in Two New Chest X-ray Databases. Sensors 2021, 21, 1742. [CrossRef]

4. Kucirka, L.M.; Lauer, S.A.; Laeyendecker, O.; Boon, D.; Lessler, J. Variation in False-Negative Rate of Reverse Transcriptase Polymerase Chain Reaction-Based SARS-CoV-2 Tests by Time Since Exposure. Ann. Intern. Med. 2020, 173, 262-267. [CrossRef]

5. Bougourzi, F.; Distante, C.; Abdelkrim, O.; Dornaika, F.; Hadid, A.; Taleb-Ahmed, A. Per-Covid-19: A Benchmark Database for Covid-19 Percentage Prediction from CT-scans. Res. Sq. 2021. [CrossRef]

6. Bougourzi, F.; Contino, R.; Distante, C.; Taleb-Ahmed, A. CNR-IEMN: A Deep Learning Based Approach to Recognise Covid-19 from CT-Scan. In Proceedings of the ICASSP 2021-2021 IEEE International Conference on Acoustics, Speech and Signal Processing (ICASSP), Toronto, ON, Canada, 6-11 June 2021; pp. 8568-8572. [CrossRef]

7. IEEE ICASSP 2021 Signal Processing Grand Challenge (SPGC) on COVID-19. Available online: http://i-sip.encs.concordia.ca/20 21SPGC-COVID19/,http:/ /i-sip.encs.concordia.ca/2021SPGC-COVID19/assets/docs/COVID-19_SPCG.pdf (accessed on 15 June 2021).

8. Afshar, P.; Heidarian, S.; Enshaei, N.; Naderkhani, F.; Rafiee, M.J.; Oikonomou, A.; Fard, F.B.; Samimi, K.; Plataniotis, K.N.; Mohammadi, A. COVID-CT-MD: COVID-19 Computed Tomography (CT) Scan Dataset Applicable in Machine Learning and Deep Learning. Sci. Data 2020, 8, 121 [CrossRef]

9. Chen, T.; Guestrin, C. Xgboost: A scalable tree boosting system. In Proceedings of the 22nd ACM Sigkdd International Conference on Knowledge Discovery and Data Mining, San Francisco, CA, USA, 13-17 August 2016; pp. 785-794.

10. Lei, J.; Li, J.; Li, X.; Qi, X. CT Imaging of the 2019 Novel Coronavirus (2019-nCoV) Pneumonia. Radiology 2020, 295, 18. [CrossRef] [PubMed]

11. Bougourzi, F.; Dornaika, F.; Mokrani, K.; Taleb-Ahmed, A.; Ruichek, Y. Fusion Transformed Deep and Shallow features (FTDS) for Image-Based Facial Expression Recognition. Expert Syst. Appl. 2020, 156, 113459. [CrossRef]

12. Paladini, E.; Vantaggiato, E.; Bougourzi, F.; Distante, C.; Hadid, A.; Taleb-Ahmed, A. Two Ensemble-CNN Approaches for Colorectal Cancer Tissue Type Classification. J. Imaging 2021, 7, 51. [CrossRef]

13. Yan, Q.; Wang, B.; Gong, D.; Luo, C.; Zhao, W.; Shen, J.; Ai, J.; Shi, Q.; Zhang, Y.; Jin, S. COVID-19 Chest CT Image Segmentation Network by Multi-Scale Fusion and Enhancement Operations. IEEE Trans. Big Data 2021, 7, 13-24. [CrossRef]

14. Müller, D.; Rey, I.S.; Kramer, F. Automated Chest CT Image Segmentation of COVID-19 Lung Infection based on 3D U-Net. arXiv 2020, arXiv:2007.04774.

15. Yao, Q.; Xiao, L.; Liu, P.; Zhou, S.K. Label-Free Segmentation of COVID-19 Lesions in Lung CT. IEEE Trans. Med. Imaging 2021. [CrossRef]

16. Alshazly, H.; Linse, C.; Barth, E.; Martinetz, T. Explainable COVID-19 Detection Using Chest CT Scans and Deep Learning. Sensors 2021, 21, 455. [CrossRef]

17. Shah, V.; Keniya, R.; Shridharani, A.; Punjabi, M.; Shah, J.; Mehendale, N. Diagnosis of COVID-19 using CT scan images and deep learning techniques. Emerg. Radiol. 2021, 28, 497-505. [CrossRef]

18. Jaiswal, A.; Gianchandani, N.; Singh, D.; Kumar, V.; Kaur, M. Classification of the COVID-19 infected patients using DenseNet201 based deep transfer learning. J. Biomol. Struct. Dyn. 2020, 1-8. [CrossRef] [PubMed]

19. Lassau, N.; Ammari, S.; Chouzenoux, e.a. Integrating deep learning CT-scan model, biological and clinical variables to predict severity of COVID-19 patients. Nat. Commun. 2021, 12, 634. [CrossRef] [PubMed] 
20. Wu, Y.H.; Gao, S.H.; Mei, J.; Xu, J.; Fan, D.P.; Zhang, R.G.; Cheng, M.M. JCS: An Explainable COVID-19 Diagnosis System by Joint Classification and Segmentation. IEEE Trans. Image Process. 2021, 30, 3113-3126. [CrossRef] [PubMed]

21. Zheng, C.; Deng, X.; Fu, Q.; Zhou, Q.; Feng, J.; Ma, H.; Liu, W.; Wang, X. Deep Learning-based Detection for COVID-19 from Chest CT using Weak Label. medRxiv 2020. [CrossRef]

22. Ter-Sarkisov, A. Covid-ct-mask-net: Prediction of covid-19 from ct scans using regional features. medRxiv 2020. [CrossRef]

23. Xue, S.; Abhayaratne, C. Covid-19 Diagnostic Using 3d Deep Transfer Learning for Classification of Volumetric Computerised Tomography Chest Scans. In Proceedings of the ICASSP 2021-2021 IEEE International Conference on Acoustics, Speech and Signal Processing (ICASSP), Toronto, ON, Canada, 6-11 June 2021; pp. 8573-8577; [CrossRef]

24. Yang, Z.; Hou, Y.; Chen, Z.; Zhang, L.; Chen, J. A Multi-Stage Progressive Learning Strategy for Covid-19 Diagnosis Using Chest Computed Tomography with Imbalanced Data. In Proceedings of the ICASSP 2021-2021 IEEE International Conference on Acoustics, Speech and Signal Processing (ICASSP), Toronto, ON, Canada, 6-11 June 2021; pp. 8578-8582; [CrossRef]

25. Chaudhary, S.; Sadbhawna, S.; Jakhetiya, V.; Subudhi, B.N.; Baid, U.; Guntuku, S.C. Detecting Covid-19 and Community Acquired Pneumonia Using Chest CT Scan Images With Deep Learning. In Proceedings of the ICASSP 2021-2021 IEEE International Conference on Acoustics, Speech and Signal Processing (ICASSP), Toronto, ON, Canada, 6-11 June 2021; pp. 8583-8587; [CrossRef]

26. Garg, P.; Ranjan, R.; Upadhyay, K.; Agrawal, M.; Deepak, D. Multi-Scale Residual Network for Covid-19 Diagnosis Using Ct-Scans. In Proceedings of the ICASSP 2021-2021 IEEE International Conference on Acoustics, Speech and Signal Processing (ICASSP), Toronto, ON, Canada, 6-11 June 2021; pp. 8558-8562; [CrossRef]

27. Diagnosing Covid-19 from CT Images Based on an Ensemble Learning Framework. In Proceedings of the ICASSP $2021-2021$ IEEE International Conference on Acoustics, Speech and Signal Processing (ICASSP), Toronto, ON, Canada, 6-11 June 2021; pp. 8563-8567; [CrossRef]

28. Lung Segmentation Tutorial. Available online: https://www.kaggle.com/ankasor/improved-lung-segmentation-usingwatershed (accessed on 15 June 2021).

29. Xie, S.; Girshick, R.; Dollár, P.; Tu, Z.; He, K. Aggregated residual transformations for deep neural networks. In Proceedings of the IEEE Conference on Computer Vision and Pattern Recognition, Honolulu, HI, USA, 21-26 July 2017; pp. $5987-5995$.

30. Huang, G.; Liu, Z.; Van Der Maaten, L.; Weinberger, K.Q. Densely connected convolutional networks. In Proceedings of the IEEE Conference on Computer Vision and Pattern Recognition, Honolulu, HI, USA, 21-26 July 2017; pp. 4700-4708.

31. Szegedy, C.; Vanhoucke, V.; Ioffe, S.; Shlens, J.; Wojna, Z. Rethinking the Inception Architecture for Computer Vision. In Proceedings of the IEEE Conference on Computer Vision and Pattern Recognition, Las Vegas, NV, USA, 26-30 June 2016; pp. 2818-2826.

32. Zagoruyko, S.; Komodakis, N. Wide Residual Networks. arXiv 2017, arXiv:1605.07146.

33. Krizhevsky, A.; Sutskever, I.; Hinton, G.E. Imagenet classification with deep convolutional neural networks. Commun. ACM 2017, 60, 84-90. [CrossRef]

34. Paszke, A.; Gross, S.; Massa, F.; Lerer, A.; Bradbury, J.; Chanan, G.; Killeen, T.; Lin, Z.; Gimelshein, N.; Antiga, L. Pytorch: An imperative style, high-performance deep learning library. Adv. Neural Inf. Process. Syst. 2019, 32, 8026-8037.

35. Lin, T.Y.; Goyal, P.; Girshick, R.; He, K.; Dollár, P. Focal loss for dense object detection. In Proceedings of the IEEE International Conference on Computer Vision, Venice, Italy, 22-29 October 2017; pp. 2980-2988.

36. Kingma, D.P.; Ba, J. Adam: A method for stochastic optimization. arXiv 2014, arXiv:1412.6980.

37. Petsiuk, V.; Das, A.; Saenko, K. RISE: Randomized Input Sampling for Explanation of Black-box Models. arXiv 2018, arXiv:1806.07421. 